\title{
Wigner-related phase spaces for signal processing and their optical implementation
}

\author{
David Mendlovic and Zeev Zalevsky \\ Faculty of Engineering, Tel Aviv University, 69978 Tel Aviv, Israel \\ Haldun M. Ozaktas \\ Faculty of Engineering, Bilkent University, 06533 Bilkent, Ankara, Turkey
}

Received March 15, 2000; revised manuscript received August 3, 2000; accepted August 3, 2000

\begin{abstract}
Phase spaces are different ways to represent signals. Owing to their properties, they are often used for signal compression and recognition with high discrimination abilities. We present several recently introduced Wigner-related sets of representations that have improved signal processing performance, and we introduce an optical implementation. This study deals with the generalized Wigner spaces, the fractional Fourier transform, and the $x-p$ and the $r-p$ representations. The optical implementations are demonstrated and discussed. (C) 2000 Optical Society of America [S0740-3232(00)02312-7]
\end{abstract}

OCIS code: 070.2590 .

\section{BACKGROUND}

\section{A. Wigner Representation}

One of the most commonly implemented phase-space representations is the Wigner distribution function ${ }^{1}$ (WDF). The WDF may be considered as a wave generalization of the Delano diagram, which is also known as the $Y \omega$ representation. The $Y \omega$ diagram is a ray model in which the $Y$ axis represents the spatial location and the $\omega$ axis represents the direction of the ray (the derivative of the first coordinate).

The WDF is useful in many fields, such as dual time frequency processing ${ }^{2}$ and data compression. ${ }^{3}$ The WDF is especially important to optics because it is a powerful tool for designing and analyzing optical systems. ${ }^{4}$ A nice example for an introduction to the WDF comes from the area of music. Neither the representation of music as a function of time nor its representation as a function of frequency is suitable for a musician. Music is displayed as a function of time and frequency (logarithmic). The musician knows at every moment what kind of sound must be produced. An extensive investigation of both the WDF and its basic properties is presented in Ref. 5 .

\section{B. Definition}

In its one-dimensional (1D) version the WDF is a mathematical operation applied on the input field distribution $u(x)$ :

$$
\begin{aligned}
\mathcal{W}\{u(x)\}= & W_{X}\left(x, f_{x}\right) \\
= & \int_{-\infty}^{\infty} u\left(x+\frac{x^{\prime}}{2}\right) u^{*}\left(x-\frac{x^{\prime}}{2}\right) \\
& \times \exp \left(-2 \pi i f_{x} x^{\prime}\right) \mathrm{d} x^{\prime} .
\end{aligned}
$$

Here $\mathcal{W}$ denotes the WDF operator and $W_{X}\left(x, f_{x}\right)$ is the Wigner chart.
Since this transform simultaneously represents spatial and spectral information of the function, it takes into account diffraction phenomena as well. For a 1D input signal, the WDF results in a two-dimensional (2D) chart presenting the spatial and the spatial spectrum information of the input (called the spatial Wigner distribution function (SWDF)].

The Wigner representation is not linear but bilinear; i.e.,

$$
\begin{aligned}
\mathcal{W}\left\{a_{1} u_{1}(x)+\right. & \left.a_{2} u_{2}(x)\right\} \\
= & \left|a_{1}\right|^{2} \mathcal{W}\left\{u_{1}(x)\right\}+\left|a_{2}\right|^{2} \mathcal{W}\left\{u_{2}(x)\right\} \\
& \quad+2 \int_{-\infty}^{\infty} \operatorname{RE}\left[a_{1} a_{2}^{*} u_{1}\left(x+\frac{x^{\prime}}{2}\right) u_{2}^{*}\left(x-\frac{x^{\prime}}{2}\right)\right] \\
& \quad \times \exp \left(-2 \pi i f_{x} x^{\prime}\right) \mathrm{d} x^{\prime} \\
\neq & a_{1} \mathcal{W}\left\{u_{1}(x)\right\}+a_{2} \mathcal{W}\left\{u_{2}(x)\right\},
\end{aligned}
$$

where $\mathrm{RE}$ is the real-part-taking operation. The reconstruction of a function from its Wigner chart can be done based on the equation

$$
u(x)=\frac{1}{u^{*}(0)} \int_{-\infty}^{\infty} W\left(\frac{x}{2}, f_{x}\right) \exp \left(2 \pi i f_{x} x\right) \mathrm{d} f_{x}
$$

Note that there is an uncertainty constant coefficient when performing the inverse WDF.

The WDF is also useful for handling optical temporal signals. Based on similar considerations, the temporal Wigner distribution function (TWDF) is defined as

$$
W_{T}\left(t, f_{t}\right)=\int_{-\infty}^{\infty} u\left(t+\frac{t^{\prime}}{2}\right) u^{*}\left(t-\frac{t^{\prime}}{2}\right) \exp \left(-2 \pi i f_{t} t^{\prime}\right) \mathrm{d} t^{\prime}
$$


where $u(t)$ is the temporal input signal and $f_{t}$ is the temporal spectrum coordinate. The inverse TWDF is defined as

$$
u(t)=\frac{1}{u^{*}(0)} \int_{-\infty}^{\infty} W\left(\frac{t}{2}, f_{t}\right) \exp \left(2 \pi i f_{t} t\right) \mathrm{d} f_{t}
$$

Present-day technology offers many possibilities for temporal optical signal processing, especially in communications applications. Several examples are demultiplexing of incoming data, ${ }^{6}$ the femtosecond pulse shaper, ${ }^{7}$ and image compression. ${ }^{8}$ Recently, space-time devices such as grating pairs, time lenses, and dispersive media were employed to design temporal signal processing systems. ${ }^{9-11}$ The TWDF may be an attractive tool for handling such systems.

\section{GENERALIZED TEMPORAL-SPATIAL WIGNER DISTRIBUTION FUNCTION}

An important family of devices and systems deals with composite spatial-temporal phenomena, for example, short pulses with a spatial distribution. Moreover, the influences of devices such as a rotated grating, which affects both the spatial and the temporal information of the signal, are impossible to represent with only the temporal or the spatial Wigner distribution chart. Owing to the increasing importance of spatial-temporal systems, ${ }^{12-14}$ a new representation that combines the temporal and the spatial information of the signal is needed. ${ }^{15}$ This new tool allows the handling of a general spatial-temporal system.

In this section we intend to present simultaneously the spatial and the temporal information of a signal, using the so-called generalized Wigner transform. ${ }^{15}$ The cases described have one spatial and one temporal dimension $[u(x, t)]$ with their Fourier conjugates $\left(f_{x}, f_{t}\right)$.

The definition of the generalized temporal-spatial Wigner distribution function (TSWDF) chart that contains four dimensions is

$$
\begin{aligned}
& W_{X T}\left(x, f_{x}, t, f_{t}\right) \\
& =\int_{-\infty}^{\infty} \int_{-\infty}^{\infty} u\left(x+\frac{x^{\prime}}{2}, t+\frac{t^{\prime}}{2}\right) u^{*}\left(x-\frac{x^{\prime}}{2}, t-\frac{t^{\prime}}{2}\right) \\
& \quad \times \exp \left(-2 \pi i f_{x} x^{\prime}\right) \exp \left(-2 \pi i f_{t} t^{\prime}\right) \mathrm{d} x^{\prime} \mathrm{d} t^{\prime} .
\end{aligned}
$$

\section{A. Properties}

Below we derive some important properties of the generalized TSWDF definition.

\section{Fourier Representation}

One can represent the signal by using its spatialtemporal Fourier representation:

$u(x, t)$

$$
=\int_{-\infty}^{\infty} \int_{-\infty}^{\infty} \tilde{u}\left(f_{x}, f_{t}\right) \exp \left(2 \pi i f_{x} x\right) \exp \left(2 \pi i f_{t} t\right) \mathrm{d} f_{x} \mathrm{~d} f_{t},
$$

which leads, according to Eq. (7), to the Fourier representation of the TSWDF:

$$
\begin{aligned}
& W_{X T}\left(x, f_{x}, t, f_{t}\right) \\
& =\int_{-\infty}^{\infty} \int_{-\infty}^{\infty} \widetilde{\hat{u}}\left(f_{x}+\frac{f_{x}^{\prime}}{2}, f_{t}+\frac{f_{t}^{\prime}}{2}\right) \widetilde{u}^{*}\left(f_{x}-\frac{f_{x}^{\prime}}{2}, f_{t}-\frac{f_{t}^{\prime}}{2}\right) \\
& \quad \times \exp \left(2 \pi i x f_{x}^{\prime}\right) \exp \left(2 \pi i t f_{t}^{\prime}\right) \mathrm{d} f_{x}^{\prime} \mathrm{d} f_{t}^{\prime} .
\end{aligned}
$$

Here $\widetilde{u}\left(f_{x}, f_{t}\right)$ represents the spatial-temporal spectrum.

One can write similar relations, but with the spatial spectrum and the temporal representation of the signal $\left[\widetilde{u}\left(f_{x}, t\right)\right]:$

$$
\begin{aligned}
& W_{X T}\left(x, f_{x}, t, f_{t}\right) \\
& =\int_{-\infty}^{\infty} \int_{-\infty}^{\infty} \tilde{u}\left(f_{x}+\frac{f_{x}^{\prime}}{2}, t+\frac{t^{\prime}}{2}\right) \widetilde{u} *\left(f_{x}-\frac{f_{x}^{\prime}}{2}, t-\frac{t^{\prime}}{2}\right) \\
& \quad \times \exp \left(2 \pi i x f_{x}^{\prime}\right) \exp \left(-2 \pi i f_{t} t^{\prime}\right) \mathrm{d} f_{x}^{\prime} \mathrm{d} t^{\prime}
\end{aligned}
$$

or with the spatial and the temporal spectrum representation $\left[\hat{u}\left(x, f_{t}\right)\right]$ :

$$
\begin{aligned}
& W_{X T}\left(x, f_{x}, t, f_{t}\right) \\
& =\int_{-\infty}^{\infty} \int_{-\infty}^{\infty} \hat{u}\left(x+\frac{x^{\prime}}{2}, f_{t}+\frac{f_{t}^{\prime}}{2}\right) \hat{u} *\left(x-\frac{x^{\prime}}{2}, f_{t}-\frac{f_{t}^{\prime}}{2}\right) \\
& \quad \times \exp \left(-2 \pi i f_{x} x^{\prime}\right) \exp \left(2 \pi i t f_{t}^{\prime}\right) \mathrm{d} x^{\prime} \mathrm{d} f_{t}^{\prime} .
\end{aligned}
$$

\section{Projections}

The projection properties of the TSWDF chart are

$$
\begin{aligned}
\int_{-\infty}^{\infty} W_{X T}\left(x, f_{x}, t, f_{t}\right) \mathrm{d} f_{x} \\
=\int_{-\infty}^{\infty} \int_{-\infty}^{\infty} u\left(x+\frac{x^{\prime}}{2}, t+\frac{t^{\prime}}{2}\right) u^{*}\left(x-\frac{x^{\prime}}{2}, t-\frac{t^{\prime}}{2}\right) \\
\quad \times \exp \left(-2 \pi i f_{t} t^{\prime}\right) \mathrm{d} x^{\prime} \mathrm{d} t^{\prime} \int_{-\infty}^{\infty} \exp \left(-2 \pi i f_{x} x^{\prime}\right) \mathrm{d} f_{x},
\end{aligned}
$$

since

$$
\int_{-\infty}^{\infty} \exp \left(-2 \pi i f_{x} x^{\prime}\right) \mathrm{d} f_{x}=\delta\left(x^{\prime}\right)
$$

Thus

$$
\begin{aligned}
\int_{-\infty}^{\infty} W_{X T}\left(x, f_{x}, t, f_{t}\right) \mathrm{d} f_{x} \\
\quad=\int_{-\infty}^{\infty} u\left(x, t+\frac{t^{\prime}}{2}\right) u^{*}\left(x, t-\frac{t^{\prime}}{2}\right) \exp \left(-2 \pi i f_{t} t^{\prime}\right) \mathrm{d} t^{\prime} \\
\quad=W_{T}\left(t, f_{t} ; x\right),
\end{aligned}
$$

where $W_{T}\left(t, f_{t} ; x\right)$ is the TWDF of $u(x, t)$, i.e., the TWDF at each location $x$. Similarly, 


$$
\begin{aligned}
\int_{-\infty}^{\infty} & W_{X T}\left(x, f_{x}, t, f_{t}\right) \mathrm{d} f_{t} \\
& =\int_{-\infty}^{\infty} u\left(x+\frac{x^{\prime}}{2}, t\right) u^{*}\left(x-\frac{x^{\prime}}{2}, t\right) \exp \left(-2 \pi i f_{x} x^{\prime}\right) \mathrm{d} x^{\prime} \\
& =W_{X}\left(x, f_{x} ; t\right),
\end{aligned}
$$

which represents the SWDF of $u(x, t)$ in a specific time $t$.

In the same manner it is easy to show that

$$
\int_{-\infty}^{\infty} \int_{-\infty}^{\infty} W_{X T}\left(x, f_{x}, t, f_{t}\right) \mathrm{d} f_{x} \mathrm{~d} f_{t}=|u(x, t)|^{2} .
$$

Now, for projections that provide spectral information,

$$
\int_{-\infty}^{\infty} W_{X T}\left(x, f_{x}, t, f_{t}\right) \mathrm{d} x=W_{T}\left(t, f_{t} ; f_{x}\right),
$$

which is the TWDF of $u(x, t)$ for a specific spatial frequency $f_{x}$. Similarly,

$$
\int_{-\infty}^{\infty} W_{X T}\left(x, f_{x}, t, f_{t}\right) \mathrm{d} t=W_{X}\left(x, f_{x} ; f_{t}\right)
$$

gives the SWDF for a specific temporal frequency. Now

$$
\int_{-\infty}^{\infty} \int_{-\infty}^{\infty} W_{X T}\left(x, f_{x}, t, f_{t}\right) \mathrm{d} x \mathrm{~d} t=\left|\tilde{\hat{u}}\left(f_{x}, f_{t}\right)\right|^{2} .
$$

Additional projection properties of the TSWDF chart are

$$
\begin{aligned}
& \int_{-\infty}^{\infty} \int_{-\infty}^{\infty} W_{X T}\left(x, f_{x}, t, f_{t}\right) \mathrm{d} f_{x} \mathrm{~d} x=W_{T}\left(t, f_{t}\right), \\
& \int_{-\infty}^{\infty} \int_{-\infty}^{\infty} W_{X T}\left(x, f_{x}, t, f_{t}\right) \mathrm{d} f_{t} \mathrm{~d} t=W_{X}\left(x, f_{x}\right), \\
& \int_{-\infty}^{\infty} \int_{-\infty}^{\infty} W_{X T}\left(x, f_{x}, t, f_{t}\right) \mathrm{d} f_{t} \mathrm{~d} x=\left|\widetilde{u}\left(f_{x}, t\right)\right|^{2}, \\
& \int_{-\infty}^{\infty} \int_{-\infty}^{\infty} W_{X T}\left(x, f_{x}, t, f_{t}\right) \mathrm{d} f_{x} \mathrm{~d} t=\left|\hat{u}\left(x, f_{t}\right)\right|^{2} .
\end{aligned}
$$

\section{Energy}

Using Eq. (15) or (18), one can easily see that

$$
\int_{-\infty}^{\infty} \int_{-\infty}^{\infty} \int_{-\infty}^{\infty} \int_{-\infty}^{\infty} W_{X T}\left(x, f_{x}, t, f_{t}\right) \mathrm{d} f_{x} \mathrm{~d} f_{t} \mathrm{~d} x \mathrm{~d} t=E_{\text {TOTAL }},
$$

where $E_{\text {TOTAL }}$ is the total energy of a signal.

\section{Spatial Lens}

If one denotes by $\hat{u}_{\mathrm{SL}}\left(x, f_{t}\right)$ the temporal spectrum distribution just after a lens of focal length $f$, then

$$
\begin{aligned}
\hat{u}_{\mathrm{SL}}\left(x, f_{t}\right) & =\hat{u}\left(x, f_{t}\right) \exp \left(\frac{-i \pi x^{2}}{\lambda f}\right) \\
& =\hat{u}\left(x, f_{t}\right) \exp \left(\frac{-i \pi f_{t} x^{2}}{c f}\right),
\end{aligned}
$$

where $c$ is light velocity and $\lambda$ is the wavelength $\left(\lambda f_{t}\right.$ $=c)$. Substituting this equation into the $W_{X T}$ definition, one obtains

$$
\begin{aligned}
W_{X T}^{\mathrm{SL}}\left(x, f_{x}, t, f_{t}\right) & \\
= & \int_{-\infty}^{\infty} \int_{-\infty}^{\infty} \hat{u}\left(x+\frac{x^{\prime}}{2}, f_{t}+\frac{f_{t}^{\prime}}{2}\right) \hat{u} *\left(x-\frac{x^{\prime}}{2}, f_{t}-\frac{f_{t}^{\prime}}{2}\right) \\
& \times \exp \left\{\frac{-i \pi\left[f_{t}+\left(f_{t}^{\prime} / 2\right)\right]\left[x+\left(x^{\prime} / 2\right)\right]^{2}}{c f}\right\} \\
& \times \exp \left\{\frac{-i \pi\left[f_{t}-\left(f_{t}^{\prime} / 2\right)\right]\left[x-\left(x^{\prime} / 2\right)\right]^{2}}{c f}\right\} \\
& \times \exp \left(-2 \pi i f_{x} x^{\prime}\right) \exp \left(2 \pi i t f_{t}^{\prime}\right) \mathrm{d} x^{\prime} \mathrm{d} f_{t}^{\prime},
\end{aligned}
$$

where $W_{X T}^{\mathrm{SL}}$ is the TSWDF obtained after the effect of the spatial lens operation. Using Eq. (17), one can easily see that the $t$-coordinate projection of the generalized STWDF, $W_{X T}^{\mathrm{SL}}$, is equal to

$$
\int_{-\infty}^{\infty} W_{X T}^{\mathrm{SL}}\left(x, f_{x}, t, f_{t}\right) \mathrm{d} t=W_{X}\left(x, f_{x}+\frac{x f_{t}}{c f} ; f_{t}\right),
$$

which is an $f_{x}$-direction shearing in the $\left(x, f_{x}\right)$ plane in which the amount of the shearing depends on the location in the $f_{t}(=c / \lambda)$ axis.

\section{Spatial Zone Plate}

Denoting by $\hat{u}_{\mathrm{ZP}}\left(x, f_{t}\right)$ the temporal spectrum distribution just after a spatial zone plate of focal length $f$, one obtains

$$
\hat{u}_{\mathrm{ZP}}\left(x, f_{t}\right)=\hat{u}\left(x, f_{t}\right) \exp \left(\frac{-i \pi f_{t_{0}} x^{2}}{c f}\right),
$$

where $f_{t_{0}}$ is the temporal frequency for which the zone plate is designed. Substituting this equation into the $W_{X T}$ definition and using Eq. (17) yields

$$
\int_{-\infty}^{\infty} W_{X T}^{\mathrm{ZP}}\left(x, f_{x}, t, f_{t}\right) \mathrm{d} t=W_{X}\left(x, f_{x}+\frac{x f_{t_{0}}}{c f} ; f_{t}\right),
$$

which is an $f_{x}$-direction shearing in the $\left(x, f_{x}\right)$ plane in which, contrary to the case of the spatial lens, the amount of the shearing does not depend on the location in the $f_{t}$ axis.

Note that the case of the spatial zone plate is different from that of the spatial lens. In the latter the $f_{x}$ shearing depends on a variable $f_{t}$, the temporal frequency of the information. In the former the $f_{x}$ shearing depends on a constant $f_{t_{0}}$, which is the temporal frequency for which the zone plate was designed.

\section{Free-Space Propagation}

A free-space propagation (FSP) module can be expressed as a multiplication of the spatial-temporal spectrum of $u(x, t)$ by a chirp:

$$
\begin{aligned}
\tilde{\hat{u}}_{\mathrm{FSP}}\left(f_{x}, f_{t}\right) & =\widetilde{\hat{u}}\left(f_{x}, f_{t}\right) \exp \left(-i \pi \lambda z f_{x}{ }^{2}\right) \\
& =\widetilde{u}\left(f_{x}, f_{t}\right) \exp \left[-i \pi c z\left(f_{x}{ }^{2} / f_{t}\right)\right],
\end{aligned}
$$

where $\widetilde{\hat{u}}_{\text {FSP }}$ is the spatial-temporal spectrum of $u$ after the effect of the FSP. $z$ is the FSP distance. Using Eq. (17), one can easily see that 


$$
\int_{-\infty}^{\infty} W_{X T}^{\mathrm{FSP}}\left(x, f_{x}, t, f_{t}\right) \mathrm{d} t=W_{X}\left(x-\frac{c z f_{x}}{f_{t}}, f_{x} ; f_{t}\right) .
$$

Thus the $t$-coordinate projection of $W_{X T}^{\mathrm{FSP}}$ is an $x$-direction shearing in the $\left(x, f_{x}\right)$ plane. Again, the amount of the shearing depends on the $f_{t}(=c / \lambda)$ value.

\section{Time Lens}

The time lens ${ }^{16}$ operation can be expressed as a multiplication of the input signal by a temporal chirp:

$$
u_{\mathrm{TL}}(x, t)=u(x, t) \exp \left[\frac{-\pi i\left(t-t_{0}\right)^{2}}{\tau^{2}}\right],
$$

where $\tau$ is the temporal focusing time and $t_{0}$ is the delay. Using the same mathematical manipulations, one can easily show that the time lens is expressed as an $f_{t}$-direction shearing in the $\left(t, f_{t}\right)$ plane:

$$
W_{X T}^{\mathrm{TL}}\left(x, f_{x}, t, f_{t}\right)=W_{X T}\left(x, f_{x}, t, f_{t}+\frac{t-t_{0}}{\tau^{2}}\right),
$$

and the $x$-coordinate projection yields

$$
\int_{-\infty}^{\infty} W_{X T}^{\mathrm{TL}}\left(x, f_{x}, t, f_{t}\right) \mathrm{d} x=W_{T}\left(t, f_{t}+\frac{t-t_{0}}{\tau^{2}} ; f_{x}\right) .
$$

Note that the device that is analogous to the time lens is the zone plate and not the spatial lens, since the shearing operation seen in Eq. (30), resembles that of Eq. (25) and not that of Eq. (23).

\section{Dispersive Medium}

A dispersion is a multiplication of the temporal spectrum by a chirp, so

$$
\hat{u}_{D}\left(x, f_{t}\right)=\hat{u}\left(x, f_{t}\right) \exp \left(-i \pi \beta f_{t}^{2}\right),
$$

where $\hat{u}_{D}$ is the temporal spectrum after the effect of the dispersion and $\beta$ is the quadratic dispersion coefficient. Equation (31) yields a shearing operation along the $t$ direction in the $\left(t, f_{t}\right)$ plane:

$$
W_{X T}^{D}\left(x, f_{x}, t, f_{t}\right)=W_{X T}\left(x, f_{x}, t-\beta f_{t}, f_{t}\right) .
$$

\section{Grating Effect}

The multiplication of the input signal by a grating function of $\exp \left(2 \pi i f_{0} x\right)$, where $f_{0}$ is the grating's frequency, provides a shift of the generalized Wigner function along the $f_{x}$ axis:

$$
W_{X T}^{G}\left(x, f_{x}, t, f_{t}\right)=W_{X T}\left(x, f_{x}-f_{0}, t, f_{t}\right) .
$$

\section{B. Analysis of a Temporal-Spatial Processor by use of the TSWDF}

In this subsection we demonstrate both the necessity and the capabilities of this new TSWDF operation. We shall analyze a temporal-spatial processor such as that illustrated in Fig. 1. ${ }^{17}$ Since the temporal input information is $1 \mathrm{D}$, it is converted into $1 \mathrm{D}$ spatial information. In the second spatial axis an imaging procedure is performed.

The suggested setup converts the temporal information of the signal into spatial information that is subsequently filtered by a spatial filter. With appropriate parameters the effect of such a filter might be temporal filtering. In this subsection we shall prove that, indeed, the first part (from the input up to the filter plane) of the setup illustrated in Fig. 1 converts the temporal information into spatial information. We use the $t$-coordinate projection of the TSWDF.

The system starts with a collimated beam (containing temporal information) that hits a grating. From Eq. (33) the $t$-coordinate projection of the TSWDF that is obtained after the grating effect is $W_{X}\left(x, f_{x}-f_{0} ; f_{t}\right)$. According to Eq. (27), beyond the FSP distance of $F$ (the focal length of the lens) the $t$-coordinate projection becomes

$$
\begin{aligned}
\int_{-\infty}^{\infty} W_{X T}\left(x, f_{x}, t, f_{t}\right) \mathrm{d} t & \\
& =W_{X}\left[x-\frac{c F\left(f_{x}-f_{0}\right)}{f_{t}}, f_{x}-f_{0} ; f_{t}\right] .
\end{aligned}
$$

After the spatial lens operation [Eq. (23)] the $t$-coordinate projection is

$$
\begin{aligned}
W_{X}[x & -\frac{c F\left(f_{x}-f_{0}\right)}{f_{t}}, f_{x}-f_{0} \\
& \left.+\frac{f_{t}}{c F}\left[x-\frac{c F}{f_{t}}\left(f_{x}-f_{0}\right)\right] ; f_{t}\right] .
\end{aligned}
$$

Additional FSP distance of $F$ gives

$$
\begin{gathered}
W_{x}\left[x-\frac{c F}{f_{t}}\left(f_{x}-f_{0}\right)-\frac{c F}{f_{t}}\left[f_{x}-f_{0}+\frac{f_{t}}{c F}\left[x-\frac{c F}{f_{t}}\left(f_{x}\right.\right.\right.\right. \\
\left.\left.\left.\left.-f_{0}\right)\right]\right], f_{x}-f_{0}+\frac{f_{t}}{c F}\left[x-\frac{c F}{f_{t}}\left(f_{x}-f_{0}\right)\right] ; f_{t}\right] .
\end{gathered}
$$

Equation (36) may be simplified to

$$
W_{x}\left[\lambda F\left(f_{0}-f_{x}\right), \frac{x}{\lambda F} ; f_{t}\right] .
$$

Let us recall that the input signal is a plane wave; thus it has no spatial information, and its spatial spectrum may be represented as $\delta\left(f_{x}\right)$. Therefore the Wigner function is sampled at $f_{x}=0$ and becomes

$$
\int_{-\infty}^{\infty} W_{X T}^{\mathrm{fin}}\left(x, f_{x}, t, f_{t}\right) \mathrm{d} t=W_{x}\left(\lambda F f_{0}, \frac{x}{\lambda F} ; f_{t}\right),
$$

where $\int_{-\infty}^{\infty} W_{X T}^{\mathrm{fin}}\left(x, f_{x}, t, f_{t}\right) \mathrm{d} t$ is the $t$-coordinate projection of the TSWDF after passage through the entire first part of the setup shown in Fig. 1 (from the input up to the filter plane). From Eq. (38) it can be seen that the spatial coordinate of the Wigner function (now denoted by $u_{x}$ ) is equal to

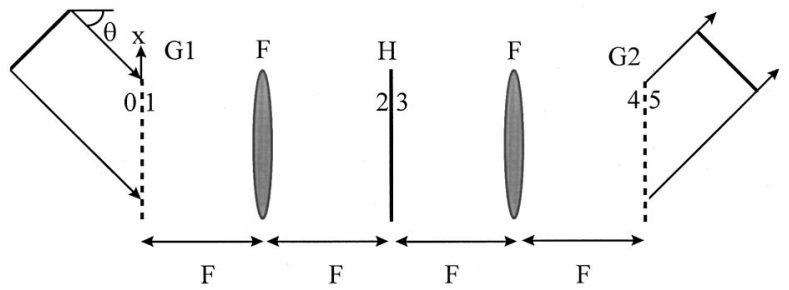

Fig. 1. Temporal-spatial processor setup. 


$$
u_{x}=\lambda F f_{0} .
$$

Thus one can see that, indeed, the spatial coordinate is proportional to the wavelength $\lambda$. Note that, if the grating is removed $\left(f_{0}=0\right)$, then $u_{x}=0$, which is a logical result inasmuch as a plane wave is focused as a spot at the origin of the focal plane of a lens. Assumption of an input wave that is not a pure plane wave will provide some distortion to the elegant expression of Eq. (38).

\section{FRACTIONAL FOURIER TRANSFORM}

The fractional Fourier transform (FRT) operation was shown to be useful for various spatial filtering and signal processing applications. ${ }^{18-27}$ The FRT is a particular case of the $A B C D$ matrix. When the $A B C D$ matrix takes the form

$$
\left[\begin{array}{ll}
A & B \\
C & D
\end{array}\right]=\left[\begin{array}{cc}
\cos \phi & -\sin \phi \\
\sin \phi & \cos \phi
\end{array}\right],
$$

the $A B C D$ transform becomes the FRT

In this transform one may control the amount of shift variance by choosing the proper fractional order $p$ for the transformation while $\phi=(p \pi) / 2$. When the fractional order is 1, the FRT becomes the conventional Fourier transform, which is totally shift invariant. For a fractional order of zero, the FRT gives the input function; i.e., the transform is totally shift variant. For any other fractional orders in between, the transform has a partial amount of shift variance.

\section{A. Definitions}

There are two common interpretations of the FRT. Both definitions were proved to be identical, as shown in Ref. 24 .

\section{Definition Based on Propagation in Graded-Index Media}

The first FRT definition ${ }^{28-30}$ is based on the field propagating along a quadratic graded-index (GRIN) medium having a length proportional to $p$ ( $p$ being the FRT order). The eigenmodes of quadratic GRIN media are the Hermite-Gaussian functions, which form an orthogonal and complete basis set. The $m$ th member of this set is expressed as

$$
\Psi_{m}(x)=H_{m}\left(\frac{\sqrt{2} x}{\omega}\right) \exp \left(-\frac{x^{2}}{\omega^{2}}\right),
$$

where $H_{m}$ is a Hermite polynomial of order $m$ and $\omega$ is a constant associated with the GRIN medium parameters. An extension to two lateral coordinates $x$ and $y$ is straightforward, with $\Psi_{m}(x) \Psi_{n}(y)$ being elementary functions.

The propagation constant for each Hermite-Gaussian mode is given by

$$
\begin{aligned}
\beta_{m} & =k\left[1-\frac{2}{k} \sqrt{\frac{n_{2}}{n_{1}}}\left(m+\frac{1}{2}\right)\right]^{1 / 2} \\
& \approx k-\sqrt{\frac{n_{2}}{n_{1}}}\left(m+\frac{1}{2}\right),
\end{aligned}
$$

with $k=2 \pi / \lambda$. The Hermite-Gaussian set is used to decompose any arbitrary distribution $u(x)$,

$$
u(x)=\sum_{m} A_{m} \Psi_{m}(x)
$$

where the coefficient $A_{m}$ of each mode $\Psi_{m}(x)$ is given by

$$
A_{m}=\int_{-\infty}^{\infty} u(x) \Psi_{m}(x) / h_{m} \mathrm{~d} x,
$$

with $h_{m}=2^{m} m ! \sqrt{\pi} \omega / \sqrt{2}$.

Using the above decomposition, we define the FRT of order $p$ as

$$
\mathcal{F}^{p}[u](x)=\sum_{m} A_{m} \Psi_{m}(x) \exp \left(i \beta_{m} p L\right),
$$

where $L=(\pi / 2) \sqrt{n_{1} / n_{2}}$ is the GRIN length that realizes the conventional Fourier transform. That this definition agrees well with the classical Fourier transform definition when $p=1$ was shown in Ref. 29 .

\section{Definition Based on Wigner Distribution Function}

In Ref. 31, Lohmann defines the FRT operation by following the signal $u(x)$ while its WDF is rotated by an angle $\phi=p \pi / 2$. Obtaining the absolute value of the signal from its Wigner distribution may be achieved by projection of the WDF onto its spectral axis. Since the Radon transform is defined as a function's projections in various angles, one may, instead of rotating the Wigner function and then projecting it onto the spectral axis, simply perform a Radon transform over the WDF in an angle corresponding to minus the desired rotation angle. Such an operation is called the Radon-Wigner transform:

$$
\left|\mathcal{F}^{p}[u](x)\right|^{2}=R_{-\phi}\{W(x, \nu)\},
$$

where $\phi=p \pi / 2$ and $R_{-\phi}\{W(x, \nu)\}$ is the Radon transform at an angle $-\phi$ of the WDF $W(x, \nu)$.

Note that the WDF of a $1 \mathrm{D}$ function is a $2 \mathrm{D}$ function and that the rotation interpretation is easily displayed. In Ref. 31 the same rotation strategy was generalized to 2D signals, i.e., images, whose WDF's are 4D distributions. The WDF of a function can be rotated with bulk optics. It was suggested in Ref. 31 that the optical system shown in Fig. 2 be used for implementation of the FRT operator.

This optical setup represents in the WDF space three shearing operations consisting of two types: $(x, \nu, x)$ shearing and $(\nu, x, \nu)$ shearing, with $\nu$ being the spectral and $x$ the spatial coordinates, respectively. The $x$ shearing is performed by FSP, then a lens performs the $\nu$ shearing, and then an $x$ shearing is again performed by additional FSP. Lohmann ${ }^{31}$ characterized this optical system by using two parameters, $Q$ and $R$ :

$$
f=f_{1} / Q, \quad z=f_{1} R,
$$

where $f_{1}$ is an arbitrary length, $f$ is the focal length of the lens, and $z$ is the distance between the lens and the input (or output) plane. As known from Ref. 31, for a FRT of order $p, Q$ and $R$ should be chosen as

$$
R=\tan (\phi / 2), \quad Q=\sin (\phi)
$$

for the type I configuration and as 


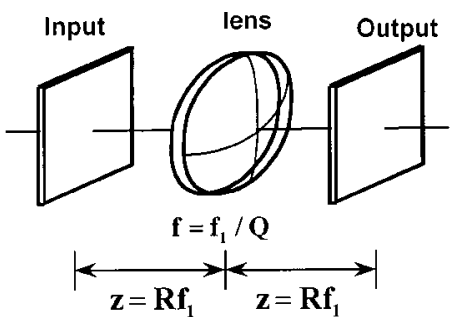

(a)

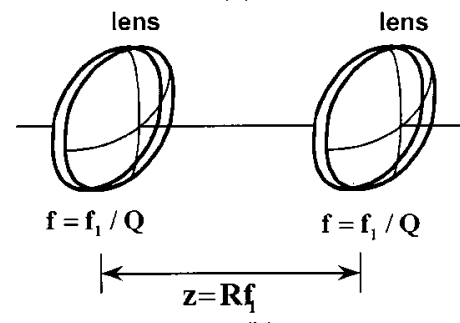

(b)

Fig. 2. The two possible optical setups for obtaining the FRT: (a) type I configuration, (b) type II configuration.

$$
R=\sin (\phi), \quad Q=\tan (\phi / 2)
$$

for the type II configuration. Note that $\phi=p(\pi / 2)$.

By analyzing the optical configuration given in Fig. 2, Lohmann ${ }^{31}$ obtained

$$
\begin{aligned}
u_{p}(x)= & \mathcal{F}^{p}\left[u\left(x_{0}\right)\right] \\
= & C_{1} \int_{-\infty}^{\infty} u\left(x_{0}\right) \exp \left(i \pi \frac{x_{0}^{2}+x^{2}}{\lambda f_{1} \tan \phi}\right) \\
& \times \exp \left(-i 2 \pi \frac{x x_{0}}{\lambda f_{1} \sin \phi}\right) \mathrm{d} x_{0},
\end{aligned}
$$

with

$$
C_{1}=\frac{\exp \left\{-i\left[\frac{\pi \operatorname{sgn}(\sin \phi)}{4}-\frac{\phi}{2}\right]\right\}}{\left|\lambda f_{1} \sin \phi\right|^{1 / 2}} .
$$

Equation (51) defines the FRT for 1D functions with $\lambda$ as a wavelength. Generalization to $2 \mathrm{D}$ functions is straightforward. Note that $\lambda f_{1}$ is also called the scaling factor.

\section{B. One Formulation for Both Definitions}

The two interpretations of the FRT operation have been united into one formulation through a transformation kernel, as illustrated in Ref. 25:

$$
u_{p}(x)=\left\{\mathcal{F}^{p}\left[u\left(x^{\prime}\right)\right]\right\}(x)=\int_{-\infty}^{\infty} B_{p}\left(x, x^{\prime}\right) u\left(x^{\prime}\right) \mathrm{d} x^{\prime},
$$

where $B_{p}\left(x, x^{\prime}\right)$ is the kernel of the transformation and $p$ is the fractional order. The kernel has two optical interpretations, one as a propagation through a GRIN medium $^{29}$ :

$$
\begin{aligned}
B_{p}\left(x, x^{\prime}\right)= & \sqrt{2} \exp \left[-\frac{1}{w}\left(x^{2}+x^{\prime 2}\right)\right] \\
& \times \sum_{n=0}^{\infty} \frac{i^{-p n}}{2^{n} n !} H_{n}\left(\frac{\sqrt{2}}{w} x\right) H_{n}\left(\frac{\sqrt{2}}{w} x^{\prime}\right),
\end{aligned}
$$

and the second as a rotation operation applied over the Wigner plane ${ }^{31}$ :

$$
B_{p}\left(x, x^{\prime}\right)=C_{1} \exp \left[i \pi\left(\frac{x^{2}+x^{\prime 2}}{\lambda f_{1} \tan \phi}\right)-2 i \pi\left(\frac{x x^{\prime}}{\lambda f_{1} \sin \phi}\right)\right] .
$$

Note that $w$ is the coefficient that connects the two interpretations:

$$
w=\sqrt{\frac{\lambda f_{1}}{\pi}}
$$

\section{RADON-WIGNER-BASED PHASE SPACES}

A. $(x, p)$ Chart

Recently, in the digital processing and the computerized tomography fields, a new tool for time frequency analysis, the Radon-Wigner transform, was suggested ${ }^{32,33}$ and was used for the time frequency representation of digital signals. ${ }^{2,34}$ This approach led to the development of a chart that contains a continuous representation of the FRT of a signal as a function of its fractional order. ${ }^{35}$ This representation may also be useful in optics, since it explicitly shows the propagation of a signal inside a GRIN medium. The approach given for producing this display starts with a 1D input signal, while the output signal contains two dimensions. The optical setup for obtaining the FRT was adapted to include only fixed FSP distances and variable lenses. With a set of two multifaceted composite holograms, the Radon-Wigner display has been experimentally demonstrated.

\section{Implementation}

We shall use the phrase $(x, p)$ display to describe a display that contains a continuous representation of a FRT of a signal as a function of the FRT order. This display may be useful both for digital signal processing (see Ref. 2) and for optics (e.g., it shows explicitly the propagation of a signal through a GRIN medium). For a 1D object, this plot contains two axes: The vertical axis is the space coordinate $x$, and the horizontal axis is the FRT order $p$. The 1D light distribution $u_{p}(x)$ [a $p$-order FRT of the original signal $u_{0}(x)$ ] is placed as a strip in the proper horizontal location in the chart according to its fractional order $p$. More explicitly, we can write

$$
F(x, p)=u_{p}(x) .
$$

As a result, all the FRT orders of the original function $u_{0}(x)$ are calculated and displayed in one plot. Figure 3 is an illustration of Eq. (56). Here the $(x, p)$ chart of the function $u(x)=\operatorname{rect}[x /(\Delta x)]$ is plotted. In the simulation we choose $\Delta x=32$ pixels. One can see that, for the cross section corresponding to $p=1$ (the Fourier transform), a $\operatorname{sinc}(\Delta x \nu)$ distribution is obtained. 
In this subsection we suggest an optical setup that optically implements the calculations of the $(x, p)$ display by a multichannel approach. The input $1 \mathrm{D}$ object is converted to a $2 \mathrm{D}$ object by use of cylindrical lenses. Then a setup that consists of a sandwich of three phase masks separated by two FSP's is constructed. The masks consist of many strips; each strip is a different channel that performs a FRT with a different order over the input signal. Each strip is a Fresnel zone plate with a different focal length that is selected for obtaining the different

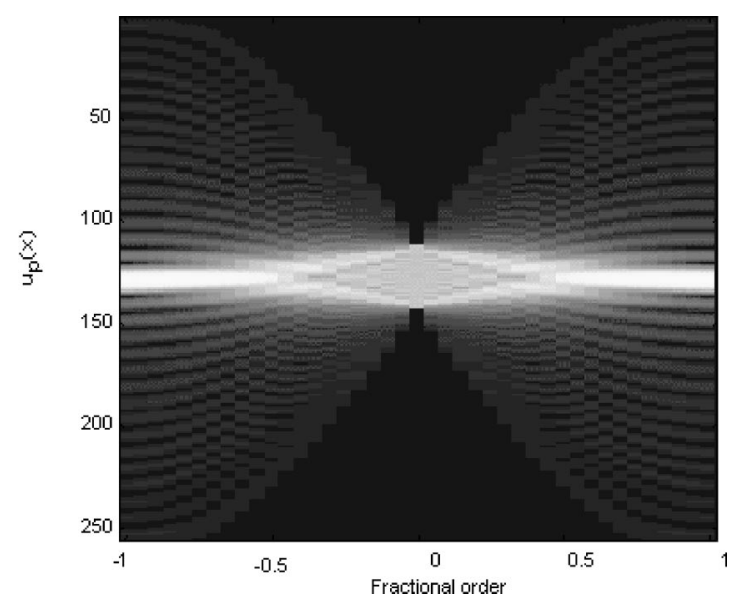

Fig. 3. Illustration of the $(x, p)$ chart.

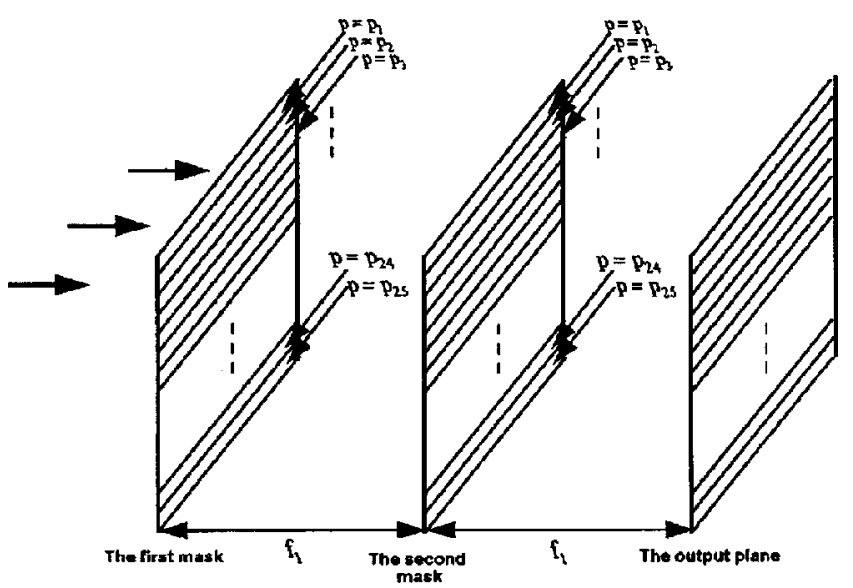

Fig. 4. Suggested optical setup for obtaining the $(x, p)$ display. fractional order $p$, and eventually the $2 \mathrm{D}$ output will be exactly the $(x, p)$ display of the $1 \mathrm{D}$ input function. Thus the first step is to prove that the setup illustrated in Fig. 4 indeed provides the FRT with different fractional orders.

Note that in this setup, whereas we are allowed to change the focal lengths (the different strips of the mask), the FSP distances are constant and remain fixed for all the fractional orders. According to Ref. 36, the optical structure given in Fig. 5(a) is totally analogous to that of Fig. 5(b) for

$$
f^{\prime}=\frac{f_{1}}{R}=\frac{f_{1}}{\sin \phi} .
$$

The proof is done with some of the Wigner optics tools. ${ }^{36}$ The tools needed are

- An inversion. This is expressed at the Wigner space as

$$
\begin{aligned}
u(x) & \rightarrow u(-x), \\
W(x, \xi) & \rightarrow W(-x,-\xi),
\end{aligned}
$$

where $x$ and $\xi$ are the two coordinates of the Wigner transform. In matrix terminology, the matrix that operates over the

$$
\left[\begin{array}{l}
x \\
\xi
\end{array}\right]
$$

vector and inverts it,

$$
\left(\left[\begin{array}{l}
-x \\
-\xi
\end{array}\right]\right)
$$

is

$$
\left[\begin{array}{cc}
-1 & 0 \\
0 & -1
\end{array}\right]
$$

This is true because

$$
\left[\begin{array}{cc}
-1 & 0 \\
0 & -1
\end{array}\right]\left[\begin{array}{l}
x \\
\xi
\end{array}\right]=\left[\begin{array}{l}
-x \\
-\xi
\end{array}\right]
$$

- Fourier transformation. This is expressed in the Wigner plane by the matrix

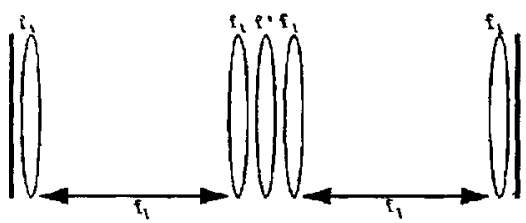

(a)

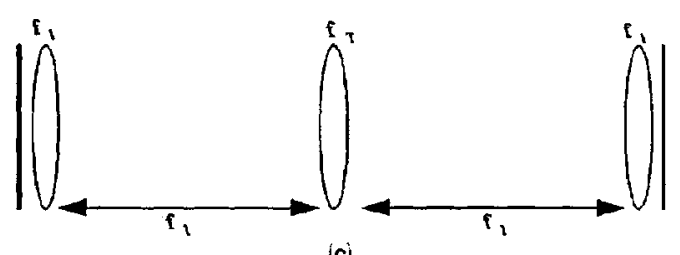

(c)

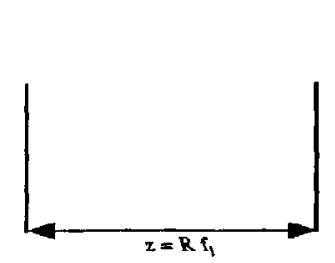

(b)

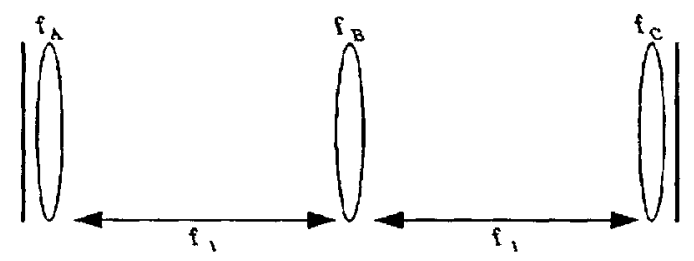

(d)

Fig. 5. The setups of (a) and (b) are totally equivalent. (c) Configuration that is equivalent to FSP of distance $z$. (d) Setup that obtains the FRT with constant distances and varying focal lengths. 


$$
\left[\begin{array}{cc}
0 & -1 \\
1 & 0
\end{array}\right]
$$

- Lens with a focal power of $R / f_{1}$ (or, mathematically, $\left.\exp \left\{-i \pi\left[\left(x^{2} R\right) / \lambda f_{1}\right]\right\}\right)$. This is expressed by the matrix

$$
\left[\begin{array}{ll}
1 & 0 \\
R & 1
\end{array}\right]
$$

- FSP over a distance of $z=R f_{1}$. This is expressed by the matrix

$$
\left[\begin{array}{cc}
1 & -R \\
0 & 1
\end{array}\right]
$$

Thus the setup described in Fig. 5(b) can be written as

$$
\left[\begin{array}{cc}
0 & -1 \\
1 & 0
\end{array}\right]\left[\begin{array}{cc}
1 & 0 \\
R & 1
\end{array}\right]\left[\begin{array}{cc}
0 & -1 \\
1 & 0
\end{array}\right]=\left[\begin{array}{cc}
0 & -R \\
0 & 1
\end{array}\right]\left[\begin{array}{cc}
-1 & 0 \\
0 & -1
\end{array}\right] .
$$

Hence a FSP of length $z=R f_{1}$ can be represented as the structure illustrated in Fig. 5(c), where

$$
f_{T}=\frac{f_{1}}{2+\sin \phi} \text {. }
$$

Applying this result to the basic FRT setup shown in Fig. 2, we replace the FSP part with the setup illustrated in Fig. 5(c). After combining the lenses' focal powers, we obtain the setup described in Fig. 5(d), with

$$
\begin{aligned}
f_{a} & =\frac{f_{1}}{\tan (\phi / 2)+1}, \\
f_{b} & =\frac{f_{1}}{\sin \phi+2}, \\
f_{c} & =f_{a} .
\end{aligned}
$$

Thus the setup suggested in Fig. 4 is appropriate for the generation of the $(x, p)$ display, since the distances of the FSP $f_{1}$ are fixed and only the focal lengths $f_{a}, f_{b}, f_{c}$ are varied according to the fractional order $p$.

Two masks that act as a varied Fresnel zone plate were constructed. These masks were generated in a multifaceted (multichannel) manner. ${ }^{37}$ Each strip (different channel) in the mask is a Fresnel zone plate with a different focal power, according to the fractional orders $p$ of the specific strip. The different focal lengths of the different strips in the first mask are related to the fractional order $p$ according to Eq. (61). In the second mask they are related according to Eq. (62). The third mask [with focal lengths according to Eq. (63)] may be placed in the output plane. This mask is unnecessary only if the absolute value of the output is examined.

The masks' function is

$$
t(x, y)=\exp (2 \pi i \alpha x) \exp \left(-\pi i \frac{x^{2}}{\lambda f}\right) \exp \left(-\pi i \frac{y^{2}}{\lambda Z_{R}}\right) .
$$

The generation of the mask was done with a computergenerated interferogram technology ${ }^{38}$ that yields a binary mask. The phase term of $\exp \left\{-\pi i\left[x^{2} /(\lambda f)\right]\right\}$ is the encoded Fresnel zone plate. $f$ is either $f_{a}$ or $f_{b}$ (depending on whether this is the first or the second mask), and it varies from one strip to the other as a function of the fractional order, as shown in Eqs. (61) and (62). The term $\exp (2 \pi i \alpha x)$ is a carrier frequency that conveys the information to the first diffraction order. To avoid overlaps among the different diffraction orders, we require

$$
\max \left|\frac{\partial \theta}{\partial x}\right|<\frac{2 \pi \alpha}{2}
$$

where $\theta=\pi x^{2} /(\lambda f)$. Thus

$$
\alpha>\left|\frac{x_{\max }}{\lambda f_{\min }}\right|,
$$

while $x_{\max }$, is the maximal $x$ coordinate and $f_{\min }$ is the minimal focal power. The term $\exp \left\{-\pi i\left[y^{2} /\left(\lambda Z_{R}\right)\right]\right\}$ was added to avoid overlapping among the different strips, which is due to diffraction. Note that in the output plane the sizes of the strips will be the same as in the first mask. This helps to avoid interference noise among the different facets. We assumed that the input wave is a Gaussian wave in its waist. $Z_{R}$ is the Rayleigh distance of the Gaussian wave and is equal to

$$
Z_{R}=\frac{\pi w^{2}}{2 \lambda},
$$

where $w$ is the waist width. Since the distance between the first and the second masks is $f_{1}$, we wish to use $Z_{R}$ $=f_{1}$.

\section{Experimental Results}

The setup suggested in Fig. 4 was constructed. The prepared masks were designed for a size of $10 \times 10 \mathrm{~mm}$ with $\lambda=532 \mathrm{~nm}$. The number of strips (channels) was 25; thus, since we assumed that the input wave was at its waist, $w=(10 \mathrm{~mm} / 25)=0.4 \mathrm{~mm} / \mathrm{channel}$, the width of the beam at the second mask is $w / \sqrt{2}$; i.e., only $1 / \sqrt{2}$ of each strip is illuminated. According to $w=0.4 \mathrm{~mm}$, one obtains $Z_{R}=472 \mathrm{~mm}$. Since in practice the input wave is not exactly at its waist, the real FSP distance should be a bit smaller than $472 \mathrm{~mm}$; thus we chose the FSP distance $f_{1}$ as $450 \mathrm{~mm}$. Since the masks sizes are 10 $\times 10 \mathrm{~mm}, x_{\max }=5 \mathrm{~mm}$. For the first mask, $f_{a_{\min }}$ (obtained for $p=1$ ) is $f_{1} / 2=225 \mathrm{~mm}$. Thus, according to inequality (66), $\alpha$ should be greater than 42 . We chose $\alpha=60$. For the second mask, the minimal power length obtained for $p=1$ is $f_{b_{\text {min }}}=f_{1} / 3=150 \mathrm{~mm}$. Thus, according to inequality (66), $\alpha>60$. Hence our choice for $\alpha$ satisfies both cases.

We produced the designed masks with a step of 0.04 in the fractional order $p$, starting from zero and ending at 0.96. Figure 6(a) illustrates the output obtained for an input of a Ronchi grating of 200 lines/cm. Figures 6(b) and 6(c) illustrate the output obtained for an input of a Ronchi grating of 100 and 50 lines/cm, respectively.

Figures 7(a) and 7(b) illustrate the output plane for an input of a chirp input $=\exp \left\{-\left[\left(i x^{2}\right) / 2 f^{2}\right]\right\}$, with the constants of $f=1.5 \mathrm{~m}$ and $f=2.5 \mathrm{~m}$, respectively.

Theoretically, it is known that the FRT of a chirp will be a delta function for the fractional order of 


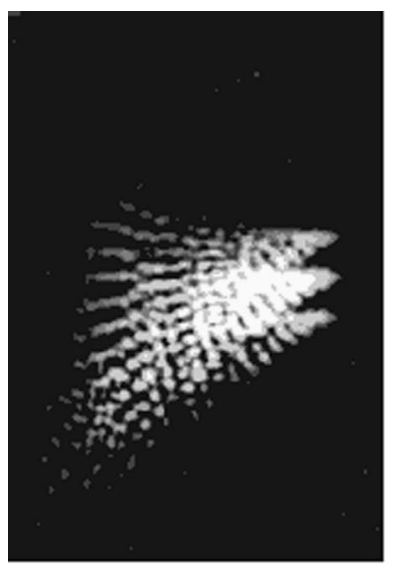

(a)

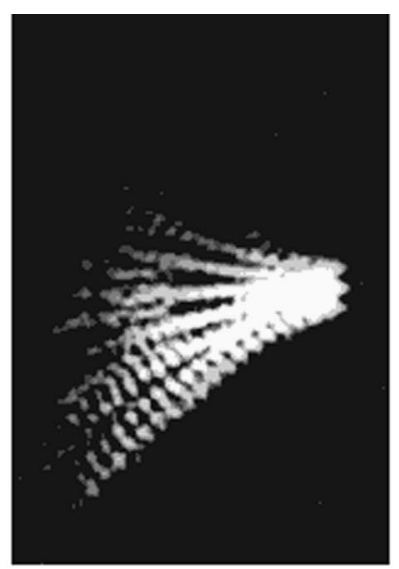

(b)

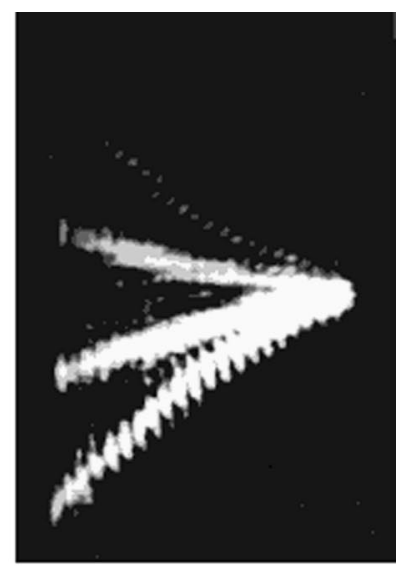

(c)

Fig. 6. Experimental results for an input of a Ronchi grating of (a) 200 lines/cm, (b) 100 lines/cm, (c) 50 lines $/ \mathrm{cm}$.

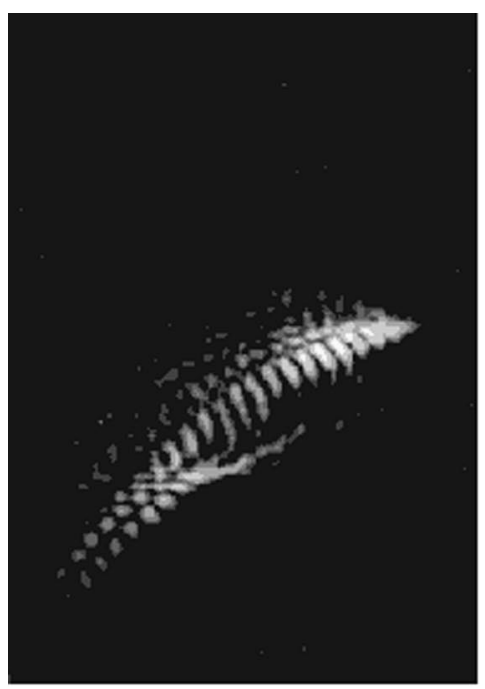

(a)

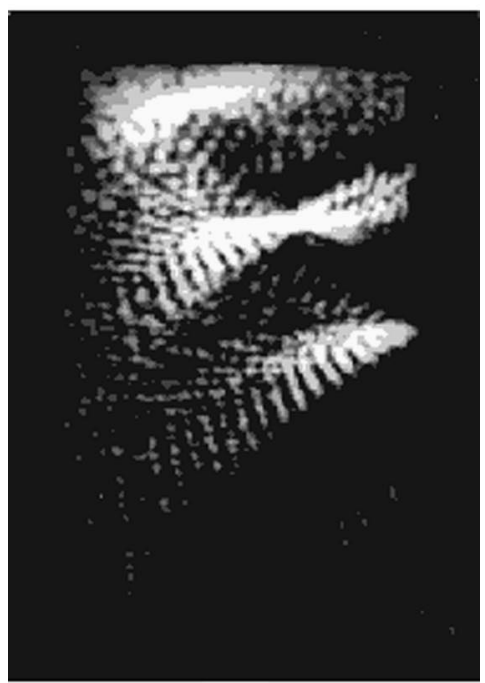

(b)

Fig. 7. Experimental results for an input of a chirp with the constants (a) $1.5 \mathrm{~m}$, (b) $2.5 \mathrm{~m}$.

$$
p=\frac{2}{\pi} \tan ^{-1}\left(\frac{2 \pi f^{2}}{\lambda f_{1}}\right)
$$

and the experimental results of Fig. 8 indeed demonstrate this effect.

\section{B. $(r, p)$ Chart}

The next step after defining the $(x, p)$ chart is to define what we call an $(r, p)$ chart. This chart performs a Cartesian-to-polar coordinate transform of the $(x, p)$ chart. ${ }^{4}$ Here all the FRT orders of the function are drawn as angular vectors. Each FRT orders is drawn along the $r$ axis in a specific angular orientation of $\phi$ $=p(\pi / 2)$, where $p$ is the fractional order. Implicitly, one can write the $(r, p)$ representation as

$$
F(r, p)=u_{p}(r) .
$$

Figure 9 is a graphical illustration of the $(r, p)$ chart representation.

It is important to note that, despite the fact that $r$ is a radial coordinate, it may obtain negative values. The $r$-coordinate negative values are a by-product of the $(r, p)$ chart definition. However, mentioning negative values for $r$ presents no conflict with the polar coordinate definition, since

$$
u_{p+2}(r)=u_{p}(-r)
$$

Another noteworthy item is associated with $r=0$. This singular point contains no relevant information and should be avoided while using the chart. As a polar representation, the required spatial resolution for a lower $r$ value is higher. Thus, in practical terms, a certain area of $|r|<r_{0}$ is not able to carry the necessary information (owing to the limited spatial resolution of every plot) and must be avoided as well.

The $(r, p)$ chart is our candidate for serving as a phasespace representation. It contains complete information about the object (along $\phi=0$ ) and about its spectrum [along $\phi=(\pi / 2)$ ]. Additional information regarding the combined space-frequency information is given along with other values of $\phi$. The inverse transformation is trivial:

$$
u_{p}(r)=F(r, p)
$$

and, for the object itself,

$$
u_{0}(r)=F(r, 0) .
$$




\section{Mathematical Properties}

Motivation. Let us recall from Lohmann ${ }^{31}$ that one can achieve the FRT by the following two algorithms:

$$
\begin{aligned}
u\left(x_{0}\right) & \Rightarrow \mathcal{W}\left\{u\left(x_{0}\right)\right\}=W(x, \nu) \Rightarrow \operatorname{Rot}\{W(x, \nu)\} \\
& \Rightarrow \text { Inverse Wigner }=u_{p}(x), \\
u\left(x_{0}\right) & \Rightarrow W(x, \nu) \Rightarrow X_{\text {shear }}\{W(x, \nu)\} \Rightarrow Y_{\text {shear }}\{W(x, \nu)\} \\
& \Rightarrow X_{\text {shear }}\{W(x, \nu)\} \Rightarrow \text { Inverse Wigner }=u_{p}(x),
\end{aligned}
$$

where Rot is the rotation operation in the plane and $X_{\text {shear }}, Y_{\text {shear }}$ are the shearing operations in the $x$ and the $y$ axes, respectively:

$$
\begin{aligned}
& X_{\text {shear }}\{f(x, y)\}=f(x+\alpha y, y), \\
& Y_{\text {shear }}\{f(x, y)\}=f(x, y+\alpha x) .
\end{aligned}
$$

Since the lens operation in the Wigner plane is a $Y_{\text {shear }}$ operation and a FSP is a $X_{\text {shear }}$ operation, the procedure described in relation (74) is in fact a FRT operation.

Note that properties very similar to those just mentioned are also relevant for the $Y \omega$ diagram. The fact that common optical operations (FSP, lens, Fourier transform, and FRT) affect the Wigner and the $Y \omega$ charts in

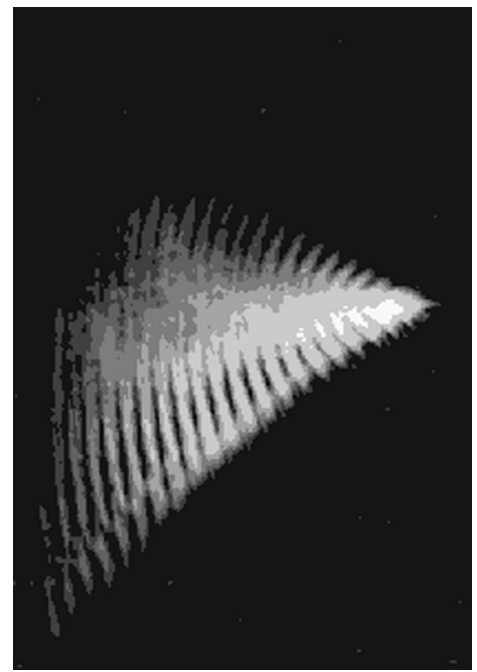

Fig. 8. Experimental results for an input of a plane wave.

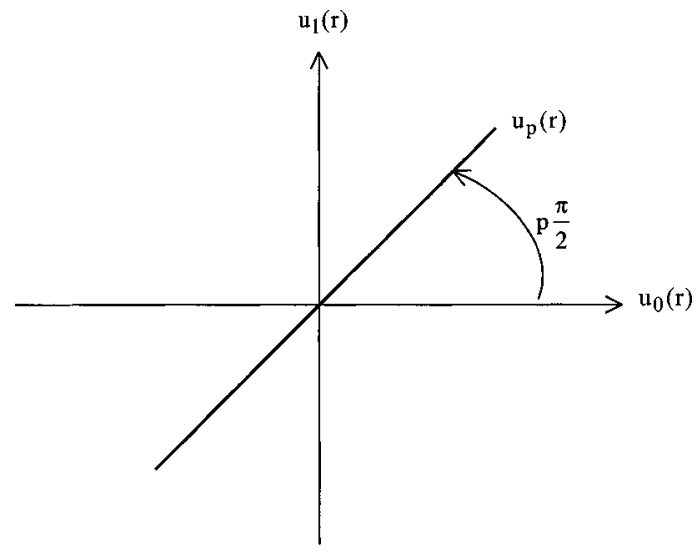

Fig. 9. Illustration of the $(r, p)$ chart. relatively simple geometrical transformations increases the potential use of these charts for analyzing and synthesizing optical systems.

Our motivation is to show that the $(r, p)$ chart has properties similar to those discussed above and that it hence might be more suitable, for some applications, than the Wigner and the $Y \omega$ charts.

Full mathematical definition. The explicit mathematical definition of the $(r, p)$ chart is based on Eqs. (50) and (70) as follows:

$$
\begin{aligned}
F(r, p)= & u_{p}(r)=C_{1} \int_{-\infty}^{\infty} u\left(x_{0}\right) \exp \left(\pi i \frac{r^{2}+x_{0}^{2}}{\tan \phi}\right) \\
& \times \exp \left(-2 \pi i \frac{r x_{0}}{\sin \phi}\right) \mathrm{d} x_{0} .
\end{aligned}
$$

Note that, in the mathematical definition of the FRT, the coordinates were normalized by $\sqrt{\lambda f_{1}}$ [in comparison with the physical definition of Eq. (50)].

To obtain the conventional Fourier transform ( $p$ $=1$ ), one should examine the distribution over the axis $\phi=\pi / 2$ on the $(r, p)$ chart. More generally, to obtain any other FRT order $p$, one should examine the chart's angular distribution at an angle of $(\pi p) / 2$.

Note that, since the FRT definition is general enough to deal with all types of signal (including complex ones), the information contained in the $(r, p)$ chart is not restricted to the type of signal.

Fractional Fourier transform operation. Assuming a function $u\left(x_{0}\right)$ and its $(r, p)$ chart $F(r, p)$, the $(r, p)$ chart of $u_{q}\left(x_{0}\right)$ [FRT of order $q$ of $u(x)_{0}$ ] is

$$
F^{q}(r, p)=\left(u_{q}\right)_{p}(r)=u_{q+p}(r)=F(r, p+q) .
$$

One can see that $F^{q}(r, p)$ is a $q \pi / 2$ angular rotation of $F(r, p)$.

Thus one can conclude that performing FRT means rotating the $(r, p)$ chart. Algorithm (73), based on the $(r, p)$ representation, is thus

$$
\begin{aligned}
u\left(x_{0}\right) & \Rightarrow F(r, p) \Rightarrow \operatorname{Rot}\{F(r, p)\} \\
& \Rightarrow \text { Inverse }(r, p) \text { chart } \Rightarrow u_{p}(x)
\end{aligned}
$$

Lens operation. One of the most common optical operators is a multiplication with a chirp function that represents a field distribution of $u_{0}\left(x_{0}\right)$ that passes through a lens. This can be written as $u_{0}\left(x_{0}\right) \exp \left(i \alpha^{\prime} \pi x_{0}^{2}\right)$ when $\alpha^{\prime}$ is related to the lens focal length $f$ as

$$
\alpha^{\prime}=-1 /(\lambda f)
$$

where $\alpha^{\prime}$ is a physical parameter whose unit is inverse square meters. Since the mathematical formulation has no unit, to use the parameter $\alpha^{\prime}$ there we define $\alpha$ $=n \alpha^{\prime}$, where $n=1\left(\mathrm{~m}^{2}\right)$.

Our interest here is to understand the effect on the $(r, p)$ chart with respect to the original chart $F(r, p)$. Let us denote the new $(r, p)$ chart as $F^{\text {lens }}(r, p)$. From Eq. (76) we can see that 


$$
\begin{aligned}
F^{(\text {lens })}(r, p)= & C_{1} \int_{-\infty}^{\infty} u_{0}\left(x_{0}\right) \exp \left(i \alpha \pi x_{0}^{2}\right) \\
& \times \exp \left(i \pi \frac{x_{0}^{2}+r^{2}}{\tan \phi}\right) \exp \left(-i 2 \pi \frac{r x_{0}}{\sin \phi}\right) \mathrm{d} x_{0} \\
= & C_{1} \exp \left(i \pi \frac{r^{2}}{\tan \phi}\right) \\
& \times \int_{-\infty}^{\infty} \exp \left[i \pi x_{0}^{2}\left(\frac{1}{\tan \phi}+\alpha\right)\right] \\
& \times \exp \left(-i 2 \pi \frac{r x_{0}}{\sin \phi}\right) \mathrm{d} x_{0} .
\end{aligned}
$$

For simplicity let us write

$$
\beta=\frac{1}{\tan \phi}+\alpha=\frac{1}{\tan \theta} .
$$

From a well-known trigonometric equation we obtain

$$
\frac{1}{\sin \theta}=\left(\beta^{2}+1\right)^{1 / 2} .
$$

Thus, based on the scale factor

$$
s=\frac{\sin \theta}{\sin \phi},
$$

Eq. (80) becomes

$$
\begin{aligned}
F^{(\text {lens })}(r, p)= & C_{1} \exp \left(i \pi \frac{r^{2}}{\tan \phi}\right) \int_{-\infty}^{\infty} u_{0}\left(x_{0}\right) \\
& \times \exp \left(i \pi \frac{x_{0}{ }^{2}}{\tan \theta}\right) \exp \left(-i 2 \pi x_{0} \frac{r s}{\sin \theta}\right) \mathrm{d} x_{0} \\
= & \psi u_{\theta}(r s),
\end{aligned}
$$

where $\psi$ is the quadratic phase factor outside the integral and $u_{\theta}(r s)$ is the $2 \theta / \pi$ FRT order of the input function with a scale factor of $s$. As a result, one can see that the effect of a lens on the $(r, p)$ chart is a coordinate transformation. Each point inside the original chart has an angular rotation and a radial scale. The rotation $\theta-\phi$ and the scale $s$ are, respectively,

$$
\begin{aligned}
\tan \theta & =\frac{\tan \phi}{1+\alpha \tan \phi}, \\
s & =\frac{1}{\sin \phi\left\{[(1 / \tan \phi)+\alpha]^{2}+1\right\}^{1 / 2}} .
\end{aligned}
$$

We dub this coordinate transformation the radial shearing transformation. The motivation for using this nickname is as follows.

After transformation to polar coordinates, Eqs. (75) become

$$
\begin{aligned}
& \theta=\tan ^{-1}\left(\frac{r \sin \phi}{r \cos \phi+\alpha r \sin \phi}\right), \\
& s=\frac{r}{\left[(r \sin \phi)^{2}+(r \cos \phi+\alpha r \sin \phi)^{2}\right]^{1 / 2}} .
\end{aligned}
$$

Division of the former equation by $r \cos \phi$ and of the latter by $r$ leads to

$$
\begin{aligned}
\tan \theta & =\frac{\tan \phi}{1+\alpha \tan \phi}, \\
s & =\frac{1}{\sin \phi\left\{1+[(1 / \tan \phi)+\alpha]^{2}\right\}^{1 / 2}} .
\end{aligned}
$$

By inspection one can see that Eqs. (85) and (87) are exactly the same, except that in Eq. (84) the scaled radius is $s r$ and in Eqs. (75) it is $r / s$. Those rotation and scale factors are dubbed the radial shearing operation. Figure 10 is a computer simulation that illustrates this new transformation operated on a rotated square.

In Fig. 10(a) the original rotated square is shown. Figures 10(b) and 10(c) show the transformed square according to the regular $X$-shearing and the radial shearing operations, respectively.

The regular $X$-shearing operation applied over a square turns it into a parallelogram.

Free-space propagation. Another important optical operation is the FSP. According to the Fresnel integral, a signal $u_{0}\left(x_{0}\right)$ that propagates through the free space along a distance $z$ is

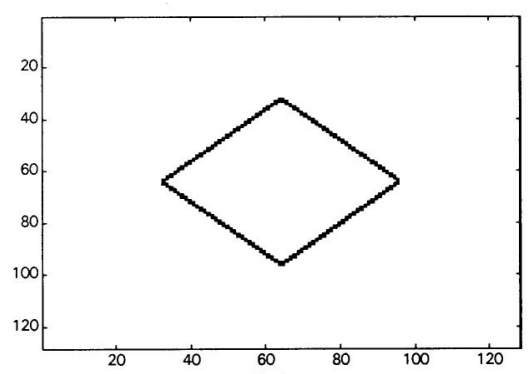

(a)

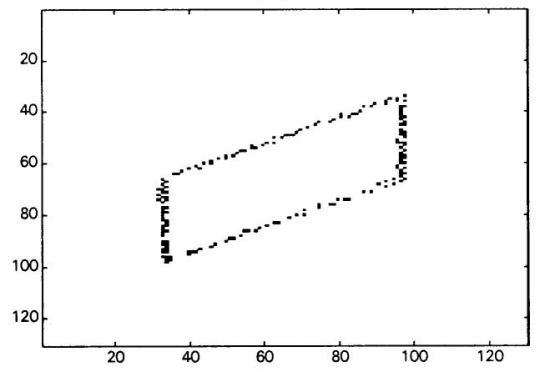

(b)

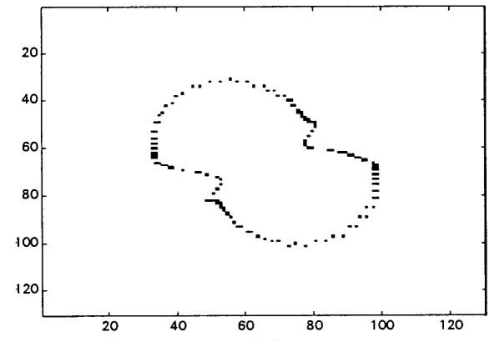

(c)

Fig. 10. (a) Rotated-square $(r, p)$ chart. (b) Its $X$-shearing transformation. (c) Its radial shearing transformation. 
$u_{i}(x, z)=\frac{\exp \left(i \frac{2 \pi}{\lambda} z\right)}{i \lambda z} \int_{-\infty}^{\infty} u_{0}\left(x_{0}\right) \exp \left[\frac{i \pi}{\lambda z}\left(x_{0}-x\right)^{2}\right] \mathrm{d} x_{0}$.

One can see that the propagation integral, which has a form of convolution, is fully equivalent to a multiplication of the spectrum of $u_{0}\left(x_{0}\right)$ by $\exp \left(-i \pi \lambda z \nu^{2}\right.$ ) (where $\nu$ is the frequency coordinate). Thus the FSP can be visualized as follows: rotation by $90^{\circ}$ of the $(r, p)$ chart, performance of a lens operation with $\alpha=-m \lambda z$ [where $m$ $=1\left(1 / \mathrm{m}^{2}\right)$ since in our mathematical formulations we want $\alpha$ to be without units], and, finally, rotation back by $-90^{\circ}$. As a result, since we have already proved that the lens operation is analogous to an $X$-shearing operation and is called radial shearing, the $90^{\circ}$ rotation will force the FSP to be analogous to the $Y$-shearing operation of the $(r, p)$ chart. This operation is dubbed the angular shearing operation.

Space-bandwidth product calculation. So far, we have investigated the effect of various optical operations on the $(r, p)$ chart. In this part we show additional information that can be extracted from the $(r, p)$ chart: the space-bandwidth product (SW) of the signal. In many cases knowledge of the SW is critical for the analysis and design of optical systems. In general, obtaining the SW is relatively complicated and involves space and frequency calculations. Using the effect of a lens and FSP on the $(r, p)$ chart, one can obtain the field distribution and the SW in every plane in the optical system. This ability gives the engineer a very powerful tool for designing and analyzing optical systems.

The SW may be defined as

$$
\mathrm{SW}=\left(\Delta F_{0}\right)\left(\Delta F_{1}\right)
$$

where $\Delta F_{p}$ is the second moment of the function $F(r, p)$ at a specific value $p(\pi / 2)$ and is defined as

$$
\Delta F_{p}=\frac{\int_{-\infty}^{\infty} r^{2}|F(r, p)|^{2} \mathrm{~d} r}{\int_{-\infty}^{\infty}|F(r, p)|^{2} \mathrm{~d} r} .
$$

Hence, after each optical element, one recalculates the $F(r, p)$ (by applying the radial and the angular shearing and rotation operations), and, from Eq. (89), the SW can easily be estimated. That is, the $\mathrm{SW}$ can be calculated at every plane of the optical system. The above definition is for the SW of the signal itself. To find the SW of other FRT orders, one can use the equation

$$
\mathrm{SW}(p)=\left(\Delta F_{p}\right)\left(\Delta F_{p+1}\right) .
$$

In several optical systems it is not necessary that $\mathrm{SW}(p)=\mathrm{SW}(0)$.

Linearity. The $F(r, p)$ chart is linear, which means that, for two (or more) different signals $u_{0}\left(x_{0}\right)$ and $v_{0}\left(x_{0}\right)$, the associated $F(r, p)$ charts may be added:

$$
F_{\text {total }}(r, p)=\alpha F_{u}(r, p)+\beta F_{v}(r, p),
$$

where $F_{\text {total }}(r, p)$ refers to the chart of $\alpha u_{0}\left(x_{0}\right)$ $+\beta v_{0}\left(x_{0}\right)$. This property does not exist in the Wigner transformation chart.

\section{Mathematical Validity}

Here several very simple optical systems are tested with the $(r, p)$ chart to examine the validity of the representation. We intend to show that elementary optical systems applied in cascade are equal to several applications of the relevant radial or angular shearing operation.

- Two lenses in cascade. It was proved that a lens operation is a radial shearing operation. Thus two lenses in cascade are equal to two radial shearing operations applied one after the other. Let us assume that a lens with a coefficient factor of $\alpha_{1}$ is applied. It has a certain radial shearing effect on the $(r, p)$ chart. Then a second lens with another coefficient factor, $\alpha_{2}$, is applied, and again another radial shearing of the $(r, p)$ chart is obtained. Here we shall prove that applying one lens with a total coefficient factor of $\alpha_{1}+\alpha_{2}$ causes a radial shearing that is equal to the overall radial shearing that was obtained above in the two-staged operation.

On the one hand, a lens with a chirp factor of $\alpha_{1}+\alpha_{2}$ provides a radial shearing of

$$
\begin{aligned}
& \theta=\tan ^{-1}\left[\frac{\tan \phi}{1+\left(\alpha_{1}+\alpha_{2}\right) \tan \phi}\right], \\
& s=\frac{1}{\sin \phi\left\{\left[(1 / \tan \phi)+\left(\alpha_{1}+\alpha_{2}\right)\right]^{2}+1\right\}^{1 / 2}} .
\end{aligned}
$$

On the other hand, applying two lens operations in cascade gives

$$
\begin{gathered}
(r, \phi) \Rightarrow\left(s_{1} r, \theta_{1}\right), \\
\left(s_{1} r, \theta_{1}\right) \Rightarrow\left(s_{2} s_{1} r, \theta\right),
\end{gathered}
$$

where

$$
\begin{aligned}
\theta_{1} & =\tan ^{-1}\left(\frac{\tan \phi}{1+\alpha_{1} \tan \phi}\right) \\
s_{1} & =\frac{1}{\sin \phi\left\{\left[(1 / \tan \phi)+\alpha_{1}\right]^{2}+1\right\}^{1 / 2}}, \\
\theta & =\tan ^{-1}\left(\frac{\tan \theta_{1}}{1+\alpha_{2} \tan \theta_{2}}\right) \\
s_{2} & =\frac{1}{\sin \theta_{1}\left\{\left[\left(1 / \tan \theta_{1}\right)+\alpha_{2}\right]^{2}+1\right\}^{1 / 2}} .
\end{aligned}
$$

After applying simple trigonometric equations as

$$
\sin ^{2} \beta=\frac{\tan ^{2} \beta}{1+\tan ^{2} \beta}
$$

one obtains, without the slightest deviation in any respect, Eqs. (93) from relations (94) and (95) when $s$ $=s_{1} s_{2}$.

- Rotation. As was mentioned above, a FRT may be obtained by use of a bulk optics systems that contain lensfree spatial lens operations. We shall show that applying the three relevant shearing operations provides precisely 
a rotation ${ }^{31}$ of the $(r, p)$ chart. This may be expected, owing to the mathematical property that FRT means a rotation of the $(r, p)$ chart.

A regular shearing operation applied over $x$ and then over $y$ and again over $x$, with factors of $A, B$, and $C$, is equivalent to

$$
\begin{aligned}
& \left(x_{0}, y_{0}\right) \Rightarrow\left(x_{0}-A y_{0}, y_{0}\right)=\left(x_{1}, y_{1}\right), \\
& \left(x_{1}, y_{1}\right) \Rightarrow\left(x_{1}, y_{1}+B x_{1}\right)=\left(x_{2}, y_{2}\right), \\
& \left(x_{2}, y_{2}\right) \Rightarrow\left(x_{2}-C y_{2}, y_{2}\right)=\left(x_{3}, y_{3}\right) .
\end{aligned}
$$

To yield a rotation by $\gamma$, the shearing coefficients should be

$$
A=C=\tan (\gamma / 2), \quad B=\sin \gamma .
$$

Now let us perform three modified shearing operations with factors of $\alpha, \beta$, and again $\alpha$, assuming that the same relation as in Eqs. (98) should be kept between the factors of the modified shearing, i.e., between $\alpha$ and $\beta$.

A modified shearing operation that is performed three times means that

$$
\begin{aligned}
(r, \phi) & \Rightarrow\left(s_{1} r, \theta_{1}\right), \\
\left(s_{1} r, \theta_{1}\right) & \Rightarrow\left(s_{1} r, \theta_{2}\right), \\
\left(s_{1} r, \theta_{2}\right) & \Rightarrow\left(s_{2} s_{1} r, \theta_{3}\right), \\
\left(s_{2} s_{1} r, \theta_{3}\right) & \Rightarrow\left(s_{2} s_{1} r, \theta_{4}\right), \\
\left(s_{2} s_{1} r, \theta_{4}\right) & \Rightarrow\left(s_{3} s_{2} s_{1} r, \theta_{5}\right),
\end{aligned}
$$

where

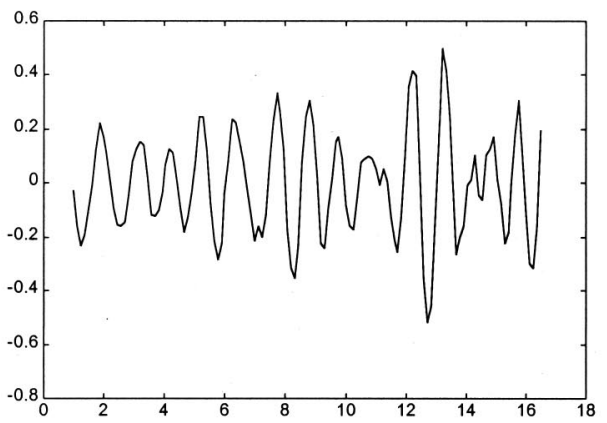

(a)

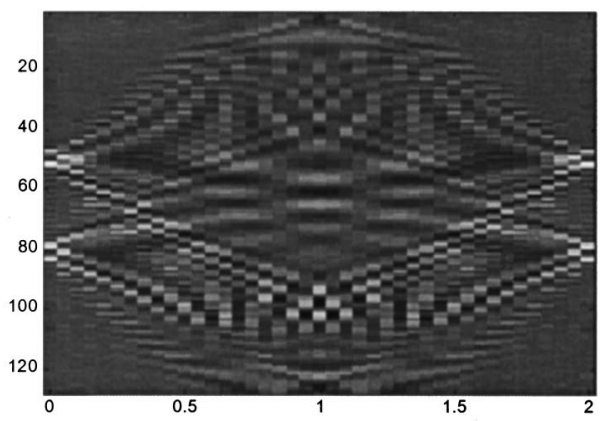

(c)

$$
\begin{aligned}
& \theta_{1}=\tan ^{-1}\left(\frac{\tan \phi}{1+\alpha \tan \phi}\right) \\
& \theta_{2}=\theta_{1}+(\pi / 2), \\
& \theta_{3}=\tan ^{-1}\left(\frac{\tan \theta_{2}}{1+\beta \tan \theta_{2}}\right), \\
& \theta_{4}=\theta_{3}-(\pi / 2), \\
& \theta_{5}=\tan ^{-1}\left(\frac{\tan \theta_{4}}{1+\alpha \tan \theta_{4}}\right), \\
& s_{1}=\frac{1}{\left.\sin \phi_{\{}[(1 / \tan \phi)+\alpha]^{2}+1\right\}^{1 / 2}}, \\
& s_{2}=\frac{1}{\sin \theta_{2}\left\{\left[\left(1 / \tan \theta_{2}\right)+\beta\right]^{2}+1\right\}^{1 / 2}}, \\
& s_{3}=\frac{1}{\sin \theta_{4}\left\{\left[\left(1 / \tan \theta_{4}\right)+\alpha\right]^{2}+1\right\}^{1 / 2}} .
\end{aligned}
$$

Note that we performed the angular shearing operation by first rotating the chart by $90^{\circ}$, then applying the radial shearing operation, and finally by again rotating the chart by $-90^{\circ}$. $\alpha$ is the radial shearing factor, and $\beta$ is the angular shearing factor. According to Eqs. (98) and the trigonometric relation

$$
\tan \frac{\gamma}{2}=\frac{\sin \gamma}{1+\left(1-\sin ^{2} \gamma\right)^{1 / 2}},
$$

we can obtain

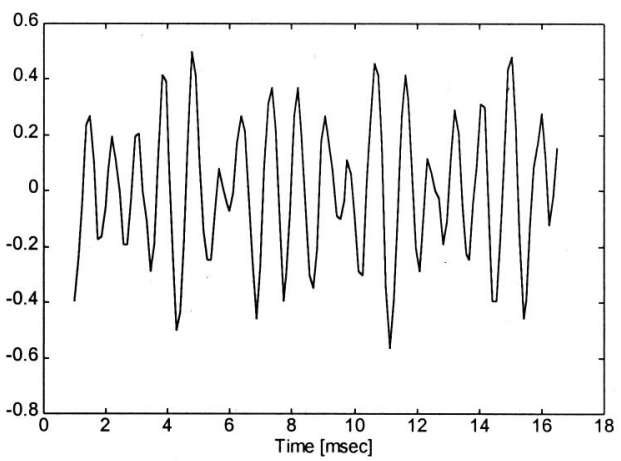

(b)

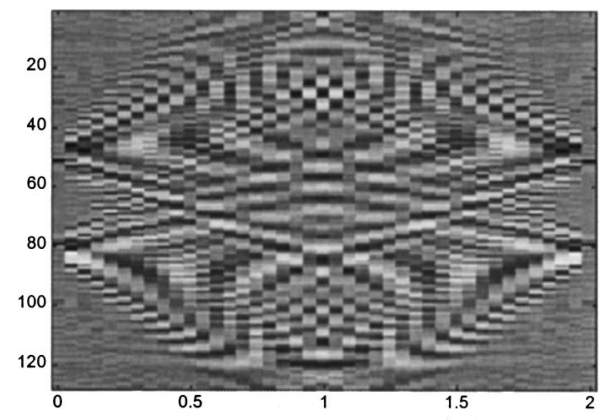

(d)

Fig. 11. (a) Reference signal $u(x)$. (b) A second, different signal $g(x)$. (c) $F_{u}(x, p)$ for the reference signal $u(x)$. (d) $F_{g}(x, p)$ for the second signal $g(x)$. 


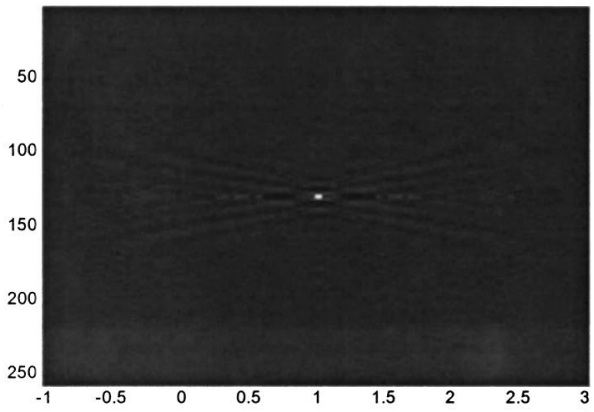

(a)

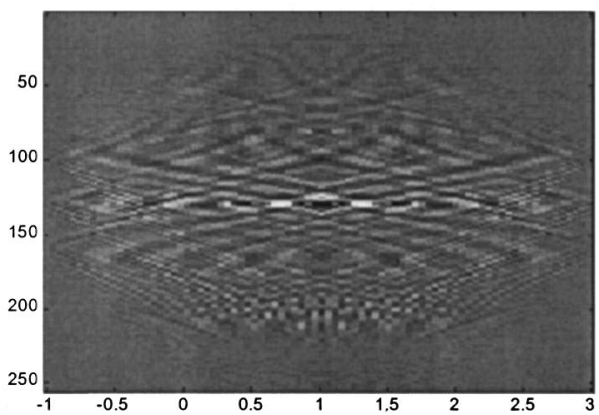

(c)

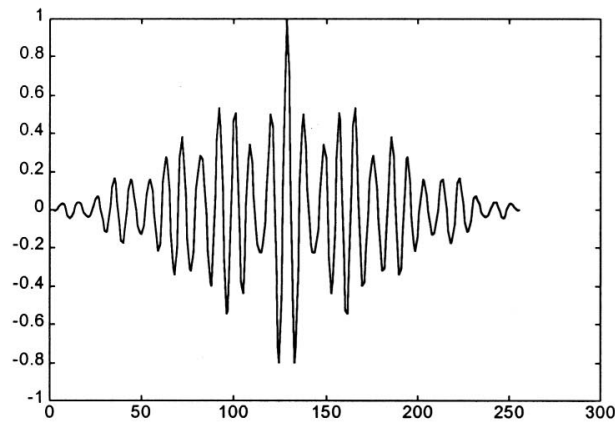

(e)

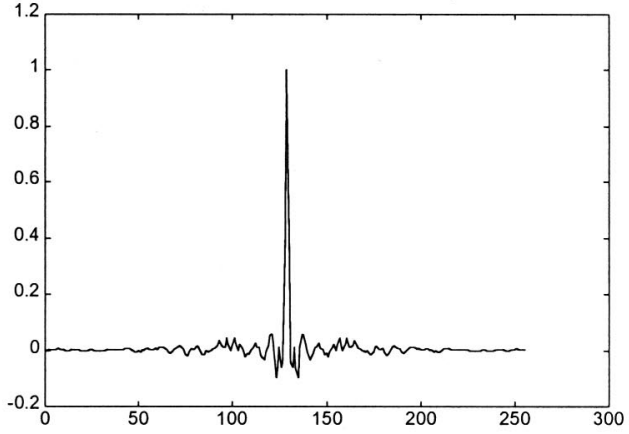

(b)

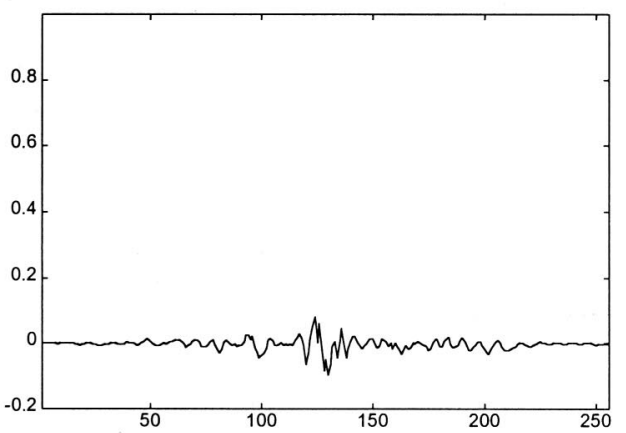

(d)

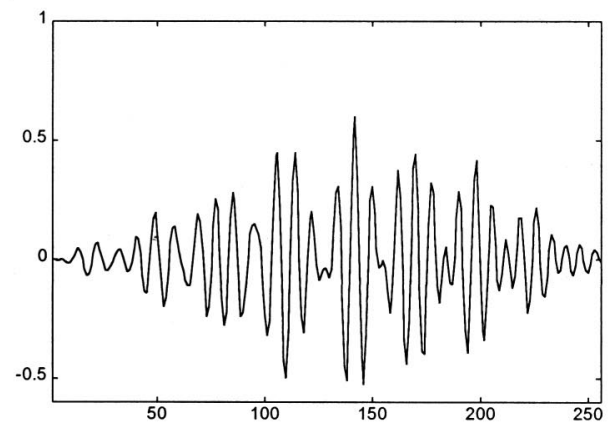

(f)

Fig. 12. (a) Correlation given by Eq. (106) when the input signal is equal to the reference signal. (b) One-dimensional cross section of (a) at $p=0$. (c) Correlation given by Eq. (106) when the input signal is equal to the second, different signal. (d) One-dimensional cross section of (c) at $p=0$. (e) Ordinary time-domain correlation of the reference signal with itself. (f) Ordinary time-domain correlation of the reference signal with the second signal.

$$
\beta=\frac{2 \alpha}{\alpha^{2}+1} .
$$

$$
\tan \left(\gamma_{1}+\gamma_{2}\right)=\frac{\tan \gamma_{1}+\tan \gamma_{2}}{1-\tan \gamma_{1} \tan \gamma_{2}}
$$

Moreover,

$$
\begin{aligned}
\frac{1}{\tan \theta_{4}} & =-\tan \theta_{3}, \\
\sin \theta_{4} & =-\cos \theta_{3}, \\
\frac{1}{\tan \theta_{2}} & =-\tan \theta_{1}, \\
\sin \theta_{2} & =\cos \theta_{1},
\end{aligned}
$$

we obtain

$$
\begin{aligned}
\theta_{5} & =\phi-\tan ^{-1} \frac{2 \alpha}{1-\alpha^{2}}, \\
s_{3} s_{2} s_{1} r & =r .
\end{aligned}
$$

Thus the radius $r$ is unchanged, and the angle is changed by $-\tan ^{-1}\left[2 \alpha /\left(1-\alpha^{2}\right)\right]$, which is precisely the definition of rotation. 


\section{Radon-Wigner Phase Spaces for Acoustic Signal Processing}

This subsection presents a new approach based on the phase-space representations introduced above. In this new technique the $(x, p)$ or the $(r, p)$ representation is correlated instead of the signals themselves.

First, the representation of a reference signal $u(x)$ is computed and stored. Then, we calculate the correlation of the incoming signal $g(x)$ with the reference, using either the Cartesian or the polar representations:

$$
\begin{aligned}
& C_{\mathrm{Car}}(x, p) \\
& \quad=\int_{-\infty}^{\infty} \int_{-\infty}^{\infty} F_{g}\left(x^{\prime}, p^{\prime}\right) F_{u} *\left(x^{\prime}-x, p^{\prime}-p\right) \mathrm{d} x^{\prime} \mathrm{d} p^{\prime},
\end{aligned}
$$

$C_{\mathrm{pol}}(x, \mu)$

$$
=\int_{-\infty}^{\infty} \int_{-\infty}^{\infty} F_{g}\left(x^{\prime}, \mu^{\prime}\right) F_{u} *\left(x^{\prime}-x, \mu^{\prime}-\mu\right) \mathrm{d} x^{\prime} \mathrm{d} \mu^{\prime},
$$

where $F_{g}\left(x^{\prime}, \mu^{\prime}\right)$ and $F_{u}\left(x^{\prime}, \mu^{\prime}\right)$ are the polar representations expressed in Cartesian coordinates $[(r, p)$ charts]. $F_{g}\left(x^{\prime}, p^{\prime}\right)$ and $F_{u}\left(x^{\prime}, p^{\prime}\right)$ are the $(x, p)$ charts.

Numerical evidence indicates that such an algorithm allows greatly superior discrimination by virtue of the additional dimension $p$ or $\mu$, as we now illustrate with a specific example.

Figure 11(a) shows a 128 pixel (15.625-ms) segment from the middle of an acoustic signal originated by a train sampled at a rate of $8192 \mathrm{~Hz}$. This segment is taken as the reference signal. For comparison, a second similar but distinct acoustic signal, shown in Fig. 11(b), was taken. Figures 11(c) and 11(d) show the Cartesian representation of the signals presented in Figs. 11(a) and 11(b), respectively. Figure 12(a) presents the autocorrelation given by Eq. (106) for the case in which the input signal is the same as the reference. Figure 12(b) presents the cross section of this correlation at $p=0$. For comparison, the correlation between the reference signal and the second signal is presented in Fig. 12(c). The cross section of this correlation, shown in Fig. 12(d), exhibits a much smaller peak. Finally, in Fig. 12(e) we show the direct ordinary time-domain correlation of the reference signal with itself, and in Fig. 12(f) we show the direct ordinary time-domain correlation of the reference signal with the second distinct signal. The peak obtained is much less distinct and highly oscillatory. Overall, it is clear that the discrimination that can be obtained from Figs. 12(e) and 12(f) is not as good as that which can be obtained from Figs. 12(b) and 12(d).

The underlying reason that the representations employed in this subsection lead to superior results may be similar to the reasons for the benefits obtained by use of wavelet transforms. In fact, a relationship between the FRT and a certain wavelet family has been pointed out in Ref. 25.

\section{Representation of Spatial-Temporal Signals}

In Subsections 4.A and 4.B we introduced the RadonWigner-based representations that we called the $(x, p)$ and the $(r, p)$ charts. Those charts were $2 \mathrm{D}$ charts for a $1 \mathrm{D}$ spatial signal. In this subsection we define more- general $(x, p)$ and $(r, p)$ displays, which are multidimensional and are defined for signals having spatial as well as temporal information. Such a general definition may be

$$
F\left(\mathbf{x}, p_{\mathbf{x}}, t, p_{t}\right)=u_{p_{\mathbf{x}}, p_{t}}(\mathbf{x}, t),
$$

where $\mathbf{x}$ is the spatial vector, $t$ is the temporal axis, and $p_{\mathbf{x}}$ and $p_{t}$ are the spatial and the temporal fractional orders, respectively.

Such a general representation may be displayed in Cartesian as well as in polar coordinate sets.

Thus this general representation can be related to the Radon transform of the generalized TS WDF.

\section{CONCLUSIONS}

In this paper we have reviewed a set of Wigner-related phase spaces that are used in various signal processing applications (such as compression and recognition with discrimination ability). Optical implementation configurations as well as experimental results or computer simulations have been presented. The transformations discussed here are the generalized Wigner transform, the fractional Fourier transform, and, primarily, the $(x, p)$ and the $(r, p)$ representations.

D. Mendlovic can be reached by e-mail at mend@ eng.tau.ac.il.

\section{REFERENCES}

1. E. Wigner, "On the quantum correction for thermodynamics equilibrium," Phys. Rev. 40, 749-752 (1932).

2. J. C. Wood and D. T. Barry, "Tomographic time-frequency analysis and its application toward time-varying filtering and adaptive kernel design for multicomponent linear-FM signals," IEEE Trans. Signal Process. 42, 2094-2104 (1994).

3. A. W. Lohmann, R. G. Dorsch, D. Mendlovic, Z. Zalevsky, and C. Ferreira, "Space-bandwidth product of optical signals and systems," J. Opt. Soc. Am. A 13, 470-473 (1996).

4. D. Mendlovic, Z. Zalevsky, R. Dorsch, Y. Bitran, A Lohmann, and H. Ozaktas, "New signal representation based on the fractional Fourier transform: definitions," J. Opt. Soc. Am. A 12, 2424-2431 (1995).

5. D. Dragoman, "The Wigner distribution function in optics and opto-electronics," in Progress in Optics, XXXVII, E. Wolf, ed. (Elsevier Science BV, Amsterdam, 1997), pp. $1-56$.

6. M. C. Nuss, M. Li, T. H. Chiu, A. M. Weiner, and Oartovi, "Time-to-space mapping of femtosecond pulses," Opt. Lett. 19, 664-666 (1994).

7. A. M. Weiner, J. P. Heritage, and E. M. Kirschner, "Highresolution femtosecond pulse shaping," J. Opt. Soc. Am. B 5, 1563-1572 (1988).

8. M. C. Nuss and R. L. Morrison, "Time-domain images," Opt. Lett. 20, 740-742 (1995).

9. Akhmanov, A. S. Chirkin, K. N. Drabovich, A. I. Kovrigin, R. V. Khokhlov, and A. P. Sukhorukov, "Nonstationary nonlinear optical effects and ultrafast light pulse formation," IEEE J. Quantum Electron. QE-4, 598-605 (1968).

10. A. W. Lohmann and D. Mendlovic, "Temporal perfectshuffle optical processor," Opt. Lett. 17, 822-824 (1992).

11. B. H. Kolner and M. Nazarathy, "Temporal imaging with a time lens," Opt. Lett. 14, 630-632 (1989). See also erratum, Opt. Lett. 15, 655 (1990).

12. W. Lukosz, "Optical systems with resolving powers exceeding the classical limit," J. Opt. Soc. Am. 56, 1463-1472 (1966). 
13. K. M. Mashoney, M. C. Nuss, and R. L. Morrison, "Terabit per second all-optical bit pattern recognition," presented at the Annual Meeting of the Optical Society of America, Portland, Oreg., September 11-15, 1995.

14. A. M. Weiner, D. E. Leaird, D. H. Retze, and E. G. Paek, "Femtosecond spectral holography," IEEE J. Quantum Electron. 28, 2251-2261 (1992).

15. D. Mendlovic and Z. Zalevsky, "Definition and properties of the generalized temporal-spatial Wigner distribution function," Optik (Stuttgart) 107, 49-56 (1997).

16. A. W. Lohmann and D. Mendlovic, "Temporal filtering with time lenses," Appl. Opt. 31, 6212-6219 (1992).

17. P. C. Sun, Y. T. Mazurenko, W. S. C. Chang, P. K. L. Yu, and Y. Fainman, "All-optical parallel-to-serial conversion by holographic spatial-to-temporal frequency encoding," Opt. Lett. 20, 1-3 (1995)

18. G. S. Agarwal and R. Simon, "A simple realization of the fractional Fourier transform and relation to harmonic oscillator Green's function," Opt. Commun. 110, 23-26 (1994).

19. L. B. Almeida, "An introduction to the angular Fourier transform," presented at the IEEE International Conference on Acoustics, Speech, and Signal Processing, ICASSP93, Minneapolis, Minn., 1993.

20. L. B. Almeida, "The fractional Fourier transform and timefrequency representations," IEEE Trans. Signal Process. 42, 3084-3091 (1994).

21. L. M. Bernardo and O. D. D. Soares, "Fractional Fourier transforms and optical systems," Opt. Commun. 110, 517522 (1994).

22. E. U. Condon, "Immersion of the Fourier transform in a continuous group of functional transformations," Proc. Natl. Acad. Sci. USA 23, 158-164 (1937).

23. R. G. Dorsch, A. W. Lohmann, Y. Bitran, D. Mendlovic, and H. M. Ozaktas, "Chirp filtering in the fractional Fourier domain," Appl. Opt. 33, 7599-7602 (1994).

24. D. Mendlovic, M. Ozaktas, and A. W. Lohmann, "Gradedindex media, Wigner-distribution functions, and the fractional Fourier transform," Appl. Opt. 33, 6188-6193 (1994).

25. H. M. Ozaktas, B. Barshan, D. Mendlovic, and L. Onural, "Convolution, filtering, and multiplexing in fractional Fourier domains and their relation to chirp and wavelet transforms," J. Opt. Soc. Am. A 11, 547-559 (1994).
26. H. M. Ozaktas, M. A. Kutay, and D. Mendlovic, "Introduction to the fractional Fourier transform and its applications," Adv. Imaging Electron Phys. 106, 239-291 (1999).

27. H. M. Ozaktas and M. A. Kutay, "Time-order signal representations," Tech. Rep. BU-CE-0005 (Department of Computer Engineering, Bilkent University, Ankara, Turkey, January 2000), presented at the First IEEE Balkan Conference on Signal Processing, Communications, Circuits, Systems, Bilkent University, Ankara, Turkey, 2000.

28. H. M. Ozaktas and D. Mendlovic, "Fractional Fourier transforms and their optical implementation. II," J. Opt. Soc. Am. A 10, 2522-2531 (1993).

29. D. Mendlovic and H. M. Ozaktas, "Fractional Fourier transforms and their optical implementation. I," J. Opt. Soc. Am. A 10, 1875-1881 (1993).

30. H. M. Ozaktas and D. Mendlovic, "Fourier transforms of fractional orders and their optical interpretation," Opt. Commun. 101, 163-165 (1993).

31. A. W. Lohmann, "Image rotation, Wigner rotation and the fractional Fourier transform," J. Opt. Soc. Am. A 10, 21812186 (1993).

32. J. C. Wood and D. T. Barry, "Radon transformation of the Wigner spectrum," in Advanced Signal Processing Algorithms, Architectures, and Implementations III, F. T. Luk, ed., Proc. SPIE 1770, 358-375 (1992).

33. A. W. Lohmann and B. H. Soffer, "Relationships between the Radon-Wigner and fractional Fourier transforms," J. Opt. Soc. Am. A 11, 1798-1801 (1994).

34. J. C. Wood and D. T. Barry, "Linear signal synthesis using the Radon-Wigner transform," IEEE Trans. Signal Process. 42, 2105-2111 (1994).

35. D. Mendlovic, R. G. Dorsch, A. W. Lohmann, Z. Zalevsky, and C. Ferreira, "Optical illustration of a varied fractional Fourier transform order and the Radon-Wigner chart," Appl. Opt. 35, 3925-3929 (1996).

36. A. W. Lohmann, "A fake zoom lens for fractional Fourier experiments," Opt. Commun. 115, 437-443 (1995).

37. H. M. Ozaktas and D. Mendlovic, "Multistage optical interconnection architectures with the least possible growth of system size," Opt. Lett. 18, 296-298 (1993).

38. W. H. Lee, "Binary synthetic holograms," Appl. Opt. 13, 1677-1682 (1974) 